\title{
Characterisation of interval-observer fault detection and isolation properties using the set-invariance approach
}

\author{
Masoud Pourasghar, Vicenç Puig*, Carlos Ocampo-Martinez \\ Universitat Politècnica de Catalunya, Institut de Robòtica i Informàtica Industrial (CSIC-UPC), Supervision, Safety \\ and Automatic Control Research Center (CS2AC), C/. Llorens i Artigas 4-6, Barcelona 08028, Spain
}

Received 20 September 2018; received in revised form 5 September 2019; accepted 7 November 2019

Available online 16 November 2019

\begin{abstract}
The aim of this paper is to provide a robust Fault Detection and Isolation (FDI) approach that combines the set-invariance approach with a zonotopic interval observer. The effect of the uncertainty is taken into account considering zonotopic-set representations in both the transient and steady states. The set-invariance approach is used to characterize the fault detectability and isolability properties in the steady-state operation of the system. In particular, the Minimum Detectable Fault (MDF) and the Minimum Isolable Fault (MIF) are characterized for several type of faults in separate formulations utilizing the integration of classical sensitivity analysis and set-invariance approaches. Finally, a simulation example based on a two-tanks system is employed to both illustrate and discuss the effectiveness of the proposed approach.
\end{abstract}

(C) 2019 The Franklin Institute. Published by Elsevier Ltd. All rights reserved.

\section{Introduction}

In order to produce efficient and high-quality products, modern processes and systems must satisfy their function correctly. Consequently, there are some important issues as safety operation, cost efficiency or environmental protection that must be considered in engineering

\footnotetext{
* Corresponding author.

E-mail address: vicenc.puig@upc.edu (V. Puig).
} 
applications [1]. In this regard, Fault Detection and Isolation (FDI) is an increasingly important area in automatic control since the fault can be known as one of the reasons of unsatisfactory performance or even instability of a dynamic system. Generally speaking, the goal of FDI theory is referred to find the root causes of the fault occurrence. After detecting the fault, maintaining the overall system stability with an acceptable performance will be the next target to be achieved by including some fault-tolerant mechanisms [2].

Model-based FDI is nowadays a well-established approach that is becoming increasingly important in the field of automatic control. Basically, model-based FDI relies on the use of a mathematical model to describe the system behavior [3,4]. The first step of FDI is Fault Detection (FD). In order to detect the fault, the real system behaviour obtained from sensors and the estimated one using the mathematical model are compared [3,5]. The fault is detected when an inconsistency is found between the real and modelled behaviours.

In model-based FDI, the performance of FD relies on improving the quality of the mathematical model [6,7]. But, due to the effect of model uncertainty, unknown disturbances and noises, the mismatch between the actual and estimated process behaviour is non-negligible even if there are no process faults [8]. Thus, consideration of the uncertainties is an important issue, and plays a key role in model-based FD framework $[9,10]$. In recent years, several methods have been proposed and developed to explicitly consider model uncertainty in FD. In particular, there exist two different paradigms for considering the uncertainty in the model. In the stochastic approaches, uncertainties are represented using random variables, while in the deterministic approaches (also called set-membership approach), uncertainties are assumed unknown but bounded by means of different type of sets, e.g., interval boxes, polytopes, ellipsoids and zonotopes [5,11-14]. According to [15], polytopes provide tighter enclosures than interval boxes. However, the main drawback of using general polytopes is related to the complexity of vertices enumeration with respect to the space dimension. But using zonotopes, basic set operations can be reduced to simple matrix calculations. This fact has recently motivated the use of zonotopes ${ }^{1}$ for modeling the effect of uncertainties [19].

Any significant inconsistency between the predicted value(s) of output(s) from the model and the real measured value(s) of output(s) given by the sensor, called residual, is known as a fault occurrence $[3,6]$. Therefore, detecting the existence of a fault relies on the comparison of the evaluated residual with a threshold value that takes into account the uncertainties $[5,20]$. In practice, the fault will be detected if the residual is larger than such a threshold. There are several approaches associated with generating the residual [3,6,21]. So far, one of the most widely used paradigms for generating the residual is the observer-based approach [22]. Observer-based approaches provide state and output estimations from the measurements and the model either stochastic (e.g., Kalman filters) or deterministic approaches (e.g., Luenberger observers) uncertainties. Then, the FD test is based on generating the residual using the output estimation error $[6,9,16,22]$.

One major issue in model-based FD framework is how to consider the effect of state disturbances, measurement noise and different faults [9,16,23-25]. Classical methods provide only an estimation based on the nominal system model. However, they do not provide a reliable characterization of the uncertainty effect in the model prediction [26]. Moreover, in the case that the residual is evaluated using statistical methods, the uncertainty is assumed to

\footnotetext{
${ }^{1}$ Zonotopes are known as a class of polytopes whose shape is implicitly represented by a rectangular matrix (see $[9,16,17]$ and [18] for further information.
} 
have known distribution. But in many cases, it is difficult to validate this assumption, where the priori knowledge of the distributions of disturbances and noises is not available [27]. Therefore, assuming that the uncertainty is unknown but bounded can be more adequate. This allows to use the set-theoretical approaches for the state estimation. Recently, there has been an increasing interest in using set-theoretical approaches in FDI, e.g., interval observer, setmembership and set-invariance approaches [9,28,29]. Among them, set-invariance approach allows to determine the residual invariant sets that can be computed in each healthy or faulty operation mode of the system [30-33]. As long as both healthy and faulty sets are separated, FDI can be performed [32,33].

One major drawback of the set-invariance approach is related to the limitation of computing the finite description of its boundary in all cases. There is a large number of published studies describing the computation of invariant sets.

The Robust Positively Invariant (RPI) set defined as a bounded region in state-space where the system state can be confined, despite of considering the bounded system uncertainties $[34,35]$. Furthermore, the minimal Robust Positively Invariant (mRPI) set is a unique and compact RPI set that contained in any closed RPI set [32,36]. In recent years, researchers have investigated a variety of approaches to construct the RPI set. So far, the proposed approaches can be classified into two main categories: (i) explicit approaches, where the RPI set is computed using the explicit formulation of the set boundary [31], (ii) iterative approaches, where the recursive iteration of the approximation of the system dynamics can be used to reach the RPI set [23,37]. When applied to FDI, the set-invariance approach is useful to check the separation of healthy and faulty residual set in the steady state.

Recently, most of the reported research about the field has highlighted the interest of using the capability of the set-invariance approach in FDI framework during the transient operation of the system using the set-theoretical approaches. In [35], the relationship between the classical observer-based and the set-invariance approaches in FD is proposed. Then, in [38-40], the characterization of the minimum magnitude of the fault that can be detected is computed by using both the observer-based and set-invariance approaches. However, there has been few discussion about the combination of the mentioned approaches. Then, both approaches are still considered as two different techniques into the FDI framework.

So far, the most serious weakness of the set-invariance approach in comparison with the interval observer approach is related to its limited use to detect faults in transient state. On the other hand, one important feature of the set-invariance approach is the ability of assessing both fault detectability and isolability properties by means of the off-line computation of the invariant sets for the residual that characterize the healthy and faulty operation modes of the system. In the set-invariance approach, the fault isolability can be obtained by guaranteeing the separability of faulty residual sets, not being possible through the use of the interval observer approach only. Therefore, the main contribution of this paper is to integrate the observer-based and set-invariance approaches to develop an FDI scheme such that it can be used in both transient and steady states of a system evolution. Furthermore, the Minimum Detectable Fault (MDF) and the Minimum Isolable Fault (MIF) are characterized based on the combination of the classical sensitivity analysis and the set-invariance approach. Moreover, the zonotopic representation of a set is considered for propagating the effect of uncertainties since its related operations can be reduced to simple matrix calculation in comparison with the huge number of vertices of the equivalent polytopes. Finally, a well-known benchmark based on the two-tank system is used as a case study for both illustrating and analyzing the effectiveness of the proposed approach in the paper. 
The structure of the paper is the following: the problem formulation is presented in Section 2. Both the zonotopic interval observer structure and its application to FD are discussed in Section 3.1. The general framework of set-invariance approach is discussed in Section 3.2. On-line propagation of the residual set and the FDI design integrating the observer-based and set-invariance approaches are presented in Section 4. In Section 5, the application of the proposed approach to a two-tank system is used in order to illustrate its effectiveness. Finally, the conclusions are drawn in Section 6. For completeness, relevant definitions and properties of zonotopes are recalled in the Appendix.

\section{Notation}

Throughout this paper, $\mathbb{R}^{n}$ denotes the set of $n$-dimensional real numbers and $\oplus$ denotes the Minkowski sum. The matrices are written using capital letter, e.g., $A$, the calligraphic notation is used for denoting sets, e.g., $\mathcal{X}$, the transfer functions are highlighted using script font e.g., $\mathscr{H},\|\cdot\|_{s}$ denotes the $s$-norm, $[\underline{x}, \bar{x}]$ is an interval with lower bound $\underline{\mathrm{x}}$ and upper bound $\bar{x}$. The notations io and $s i$ in the mathematical formulations denote the interval observer and set-invariance approaches, respectively.

\section{Problem formulation}

\subsection{Problem set-up}

In this paper, discrete-time linear uncertain system to be monitored is described in state space as

$x_{k+1}=A x_{k}+B u_{k}+E_{\omega} \omega_{k}$,

$y_{k}=C x_{k}+E_{v} v_{k}$,

where $u \in \mathbb{R}^{n_{u}}, y \in \mathbb{R}^{n_{y}}$ and $x \in \mathbb{R}^{n_{x}}$ are the input, the output and the state vectors, respectively. Moreover, $A \in \mathbb{R}^{n_{x} \times n_{x}}, B \in \mathbb{R}^{n_{x} \times n_{u}}$ and $C \in \mathbb{R}^{n_{y} \times n_{x}}$ are the state-space matrices. Both state disturbance and process noise vectors are defined by $\omega \in \mathbb{R}^{n_{x}}$ and $v \in \mathbb{R}^{n_{y}}$, respectively. Moreover, $E_{\omega}$ and $E_{v}$ are the associated distribution matrices with appropriate dimensions while $k \in \mathbb{N}$ indicates the discrete time.

Due to the effect of the process disturbance and measurement noise in Eq. (1), a perfectly accurate and complete of the estimated behaviour of the systems is not possible. Therefore, the effect of modeling uncertainties in Eq. (1) is one of the most crucial points in this paper. In this regard, the considered uncertainties are assumed to be unknown but bounded in their uncertainty intervals and can vary at each time step, i.e., $\omega_{k} \in \mathcal{W}_{k}$ and $v_{k} \in \mathcal{V}_{k}$, where $\mathcal{W}_{k}$ and $\mathcal{V}_{k}$ are interval boxes

$\mathcal{W}_{k}=\left\{\omega_{k} \in \mathbb{R}^{n_{x}}:\left|\omega_{k}-c_{\omega, k}\right| \leq \bar{\omega}_{k}, c_{\omega, k} \in \mathbb{R}^{n_{x}}, \bar{\omega}_{k} \in \mathbb{R}^{n_{x}}\right\}$

$\mathcal{V}_{k}=\left\{v_{k} \in \mathbb{R}^{n_{y}}:\left|v_{k}-c_{v, k}\right| \leq \bar{v}_{k}, c_{v, k} \in \mathbb{R}^{n_{y}}, \bar{v}_{k} \in \mathbb{R}^{n_{y}}\right\}$,

where $c_{\omega}, \bar{\omega}, c_{v}$ and $\bar{v}$ are constant vectors. As mentioned before, the effect of uncertainty can be characterized using a zonotopic-set representation (i.e., a particular type of polytope) 
reducing the set operations to simple matrix calculations [29,41]. In this regard, the zonotopic representation of $\omega$ and $v$ in Eq. (2) can be written as

$\mathcal{W}=\left\langle c_{\omega}, R_{\omega}\right\rangle$

$\mathcal{V}=\left\langle c_{v}, R_{v}\right\rangle$

where $c_{\omega}$ and $c_{v}$ denote the centers of the measurement disturbance and the process noise zonotopes, respectively, with their generator matrices $R_{\omega} \in \mathbb{R}^{n_{\omega} \times n_{\omega}}$ and $R_{v} \in \mathbb{R}^{n_{v} \times n_{v}}$.

Assumption 2.1. The additive uncertainties represented in Eq. (3) are assumed to be bounded by a unit hypercube expressed as the centered zonotopes, i.e., for all $k \geq 0, \omega \in\left\langle 0, I_{n_{\omega}}\right\rangle$ and $v \in\left\langle 0, I_{n_{v}}\right\rangle$, where $I_{n_{\omega}} \in \mathbb{R}^{n_{\omega} \times n_{\omega}}$ and $I_{n_{v}} \in \mathbb{R}^{n_{v} \times n_{v}}$ denote the identity matrices.

Assumption 2.2. The initial state $x_{0}$ belongs to the zonotopic set $\mathcal{X}_{0}=\left\langle c_{0}, R_{0}\right\rangle$, where $c_{0} \in$ $\mathbb{R}^{n_{x}}$ denotes the center and $R_{0} \in \mathbb{R}^{n_{x} \times r_{R_{0}}}$ is a non-empty matrix containing the generators matrix of the initial zonotope $\mathcal{X}_{0}$.

From hereafter, the subscript $k+1$ will be replaced by + and $k$ will be omitted for the sake of simplified notations. Consequently, the dynamical model (1) can be written as

$x_{+}=A x+B u+E_{\omega} \omega$,

$y=C x+E_{v} v$.

Furthermore, for the sake further simplification, the acronyms IOA and SIA will be used to denote the interval-observer and set-invariance approaches, respectively.

\subsection{Observer structure}

Monitoring the healthy operation of a system with the dynamical model (4) can be done by designing a linear Luenberger observer of the form

$\hat{x}_{+}=A \hat{x}+B u+L(y-\hat{y})$,

$\hat{y}=C \hat{x}$,

where $\hat{x}$ and $\hat{y}$ are the state estimation and the output prediction, respectively. Furthermore, $L$ denotes the observer gain that should be chosen such $(A-L C)$ is a Schur matrix (i.e., with all eigenvalues with a module less than unity) that guarantees the convergence of the observer. Moreover, $L$ can provide degrees of freedom to tune the system monitoring, e.g., with the goal of optimizing the detection of faults. Moreover, the pair $\{A, C\}$ is assumed to be detectable.

\section{Preliminaries}

\subsection{Zonotopic interval-observer approach}

Using the zonotopic-set representation of the uncertainties, i.e., $\mathcal{W}$ and $\mathcal{V}$, the state bounding observer for the dynamical model (4) can be obtained as a zonotope $\hat{\mathcal{X}}=\langle c, R\rangle$ using the Luenberger observer (5) and Proposition 3.1. 
Proposition 3.1 (Zonotopic observer structure). Considering Assumptions 2.1 and 2.2 and the observer (5), the center $c$ and the shape matrix $R$ of $\hat{\mathcal{X}}$ can be recursively computed as

$c_{+}=(A-L C) c+B u+L y$,

$R_{+}=\left[\begin{array}{ll}(A-L C) \bar{R} & E_{d}\end{array}\right]$,

where $E_{d}=\left[E_{\omega}, \quad-L E_{v}\right]$ and $\bar{R}=\downarrow_{q}\{R\}$. Moreover, the state inclusion property $x \in\langle c, R\rangle$ in Properties 3 and 4 holds for all $k \geq 0$.

Proof. Assume $x \in\langle c, R\rangle, \omega \in\left\langle 0, I_{n_{\omega}}\right\rangle$ and $v \in\left\langle 0, I_{n_{v}}\right\rangle$ for all $k \geq 0$, where the inclusion property is preserved by using the reduction operator, i.e., $x \in\langle c, \bar{R}\rangle$. Thus, the state observer in Eq. (5a) can be written using the set representation as

$$
x_{+} \in\left\langle c_{+}, R_{+}\right\rangle=\langle(A-L C) c,(A-L C) \bar{R}\rangle \oplus\left\langle B_{u} u, 0\right\rangle \oplus\left\langle 0, E_{\omega}\right\rangle \oplus\langle L y, 0\rangle \oplus\left\langle 0,-L E_{v}\right\rangle .
$$

Then, based on Definition 3 and Property 1, $c_{+}$and $R_{+}$in Eq. (7) can be expressed as in Eq. (6).

Consequently, considering Proposition 3.1 and assuming $x \in\langle c, R\rangle$ and $v \in\left\langle 0, I_{n_{v}}\right\rangle$ for all $k \geq 0$, the output prediction vector $y$ in Eq. (5b) satisfies $y \in\left\langle c_{y}, R_{y}\right\rangle=\langle C c, C \bar{R}\rangle \oplus\left\langle 0, E_{v}\right\rangle$. Thus, in healthy system operation, it can be written that

$c_{y}=C c$,

$R_{y}=\left[\begin{array}{ll}C \bar{R} & E_{v}\end{array}\right]$

Mainly, a fault can be detected by generating the residual $r=y-\hat{y}$. Therefore, using Eq. (4b) and (8), the residual zonotope can be computed as

$c_{r^{i o}}=y-C c$,

$R_{r^{i o}}=\left[\begin{array}{ll}-C \bar{R} & -E_{v}\end{array}\right]$.

Hence, the FD test is based on checking the satisfaction of $0 \notin\left\langle c_{r^{i o}}, R_{r^{i o}}\right\rangle$. The computational burden can be reduced to check whether 0 is inside or not of an aligned box enclosing the zonotope $\left\langle c_{r^{i o}}, R_{r^{i o}}\right\rangle$ as

$0 \notin\left\langle c_{r^{i o}}, b\left(R_{r^{i o}}\right)\right\rangle$,

where $\left\langle c_{r^{i o}}, b\left(R_{r^{i o}}\right)\right\rangle$ is enclosed by an aligned box characterized as $b\left(R_{r^{i o}}\right)=\operatorname{diag}\left(\left|R_{r^{i o}}\right| \mathbf{1}\right)$. Considering that $|$.$| is the element-by-element absolute value operator, \mathbf{1}$ is a column vector of ones and $\operatorname{diag}($.) returns a diagonal matrix from a vector of diagonal elements. In the case of satisfaction of Eq. (10), the existence of the fault will be detected. Otherwise, the system is considered healthy.

\subsection{Set-invariance approach}

Generally speaking, given the system (4) and considering the observer (5), the trajectories of the residual will ultimately converge to an invariant set. Then, based on [32] and [29], the constructed RPI set in state space can be projected to the residual space and whenever the 
corresponding residual is inside this set, it will remain inside. Furthermore, the residual can be generated as

$r=y-\hat{y}=C \tilde{x}+E_{v} v$,

where $\tilde{x}=(x-\hat{x})$ is the state estimation error whose dynamics can be described using Eqs. (4) and (5) as

$\tilde{x}_{+}=(A-L C) \tilde{x}+E_{d} d$,

where $d=\left[\begin{array}{ll}\omega & v\end{array}\right]^{T}$.

According to [42], the set $\Phi^{\tilde{x}}$ is an RPI set for Eq. (12) if and only if, for all $\omega \in \mathcal{W}$ and $v \in \mathcal{V}$

$(A-L C) \tilde{x}+E_{d} d \in \Phi^{\tilde{x}}$.

There is a large amount of reported results describing the construction of $\Phi^{\tilde{x}}$ [30]. In this paper, the Ultimate Bound (UB) method reported in [31] will be used in order to obtain the RPI set. Therefore, $\Phi^{\tilde{x}}$ can be computed using Theorem 3.1.

Theorem 3.1. [31] consider the Jordan Conical form of matrix $(A-L C)$ as $J=V(A-$ $L C) V^{-1}$, where $J$ is a diagonal matrix corresponding to the Jordan-normal form of (A$L C)$ and $V$ is a non-singular transformation matrix. Thus, the state estimation error $\tilde{x}$ in Eq. (12) will ultimately converge within the polyhedral RPI set that is constructed as

$\Phi^{\tilde{x}}=\left\{\tilde{x} \in \mathbb{R}^{n_{x}}:\left|V^{-1} \tilde{x}\right| \leq(I-|J|)^{-1}\left|V^{-1}\right| \bar{d}+\varepsilon\right\}$,

where $\varepsilon$ can be any arbitrary small vector with strictly positive components.

Therefore, $\Phi^{\tilde{x}}$ can be computed straightaway using Theorem 3.1. Then, considering $\tilde{x} \in \Phi^{\tilde{x}}$ and $v \in \mathcal{V}$, the projection of $\tilde{x}$ to a residual space, i.e., invariant set for the residual in Eq. (11) denoted by $\Phi^{r}$, can be computed as

$\Phi^{r}=C \Phi^{\tilde{x}} \oplus \mathcal{V}$.

According to [35], if $\tilde{x} \in \Phi^{\tilde{x}}$, then $r \in \Phi^{r}$. Therefore, the existence of the fault will be detected based on the SIA whenever the satisfaction of the following condition is proved:

$r \notin \Phi^{r}$.

\section{Characterization of Detectability and Isolability Properties}

The main objective of this section is to combine IOA and SIA in order to exploit their benefits and overcome their drawbacks. In particular, IOA will provide an on-line test that can be applied in both transient and steady states response and SIA will allow to characterize detectability and isolability properties in the steady state.

\subsection{On-line propagation of the residual set}

Given the observer (5) and considering unknown but bounded uncertainties, two main approaches are presented in this paper to detect the fault: (i) IOA and ii) SIA. Each of them has its own advantages and drawbacks. In the former approach, the FD principle leads to detect the fault in both transient and steady-state operation of the system since its residual 
generation is performed on-line. The latter approach only works in steady state since its residual generation is performed off-line.

Therefore, the most serious weakness of the SIA in comparison with the IOA is related to its FDI limitation in transient state. On the other hand, one important feature of SIA is the ability of providing both detectability and isolability properties in comparison with IOA. Therefore, the purpose of this paper is to propose an FDI approach based on IOA that integrates IOA and SIA that can

- be used during the whole time range (both transient and steady states), and

- guarantee both detectability and isolability properties.

According to [37] and [23], the RPI set in SIA (see Section 3.2), can be alternatively computed by recursive iteration of $\hat{x}$. Thus, the dynamical model (4) and the zonotopic state bounding observer in Proposition 3.1 can be used to obtain the zonotopic representation of the state estimation error and consequently the RPI set.

Coming back to the main issue discussed at the beginning of this section, it is now time to compute the RPI set of the state estimation error. In this regard, Proposition 4.1 implies that $\Phi^{\tilde{x}}$ can also be represented as a zonotope.

Proposition 4.1 (Zonotopic representation of the RPI set for $\tilde{x}$ ). Consider a state estimation error dynamics in Eq. (12), the zonotopic RPI set of the state estimation error can be computed as

$c_{\tilde{x}_{j+1}}=(A-L C) c_{\tilde{x}_{j}}$,

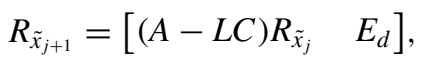

where $j \in \mathbb{N}$ denotes the $j$ th element of the set. Then, in the steady state, i.e., when $j \rightarrow \infty$, it can be written that

$c_{\tilde{x}_{\infty}}=0$,

$\left\|R_{\tilde{x}_{\infty_{i}}}\right\|_{1}=\left\|R_{\infty_{i}}\right\|_{1}$,

where $i$ denotes the ith row of the matrices.

Proof. Consider the dynamical model of the state estimation error in (12) and assume that the initial state estimation error $\tilde{x}_{0}$ belongs to the zonotopic set $\tilde{\mathcal{X}}_{0}=\left\langle c_{\tilde{x}_{0}}, R_{\tilde{x}_{0}}\right\rangle$ that is defined as an RPI set, since $\tilde{x} \in\left\langle c_{\tilde{x}}, R_{\tilde{x}}\right\rangle, \omega \in\left\langle 0, I_{n_{\omega}}\right\rangle$ and $v \in\left\langle 0, I_{n_{v}}\right\rangle$ for all $k \geq 0$, it can be written that

$\tilde{x}_{j+1} \in\left\langle c_{\tilde{x}_{j+1}}, R_{\tilde{x}_{j+1}}\right\rangle=\left\langle(A-K C) c_{\tilde{x}_{j}},(A-K C) R_{\tilde{x}_{j}}\right\rangle \oplus\left\langle 0, E_{\omega}\right\rangle \oplus\left\langle 0,-K E_{v}\right\rangle$

is another RPI set with arbitrarily expected precision enclosing the mRPI set of the state estimation error in Eq. (12). Thus, the center and the shape matrix of the set in Eq. (19) can be unfolded as in Eq. (17).

Furthermore, the state estimation error will converge to the RPI set in steady state. Thus, the RPI set of $\tilde{x}$ can be computed by recursive propagation of the zonotopic set (17) starting from the initial set $\tilde{\mathcal{X}}_{0}=\left\langle c_{\tilde{x}_{0}}, R_{\tilde{x}_{0}}\right\rangle$ that belongs to the RPI set which can be computed using 
the any available method such as UB method recalled in Section 3.2. Furthermore, it can be stated that if $j \rightarrow \infty$ (i.e., in steady state), the following conditions will be satisfied:

$c_{\tilde{x}_{j+1}}=c_{\tilde{x}_{j}}$,

$\left\|R_{\tilde{x}_{j+1}}\right\|_{1}=\left\|R_{\tilde{x}_{j}}\right\|_{1}$.

Therefore, the same formulations as Eq. (18) for the center and the shape matrix of $\tilde{\mathcal{X}}$ can be obtained by substitution of conditions (20) in (17).

Now, considering Proposition 4.1, the zonotopic representation of the residual set in steady state can be computed by computing the projection of $\tilde{x}$ into the residual space as

$c_{r^{s i}}=C c_{\tilde{x}_{\infty}}$,

$R_{r^{s i}}=\left[\begin{array}{ll}C R_{\tilde{x}_{\infty_{i}}} & E_{v}\end{array}\right]$

Therefore, considering Proposition 4.1, the residual set in steady state is invariant and can be considered as a set that combines the polytopic UB method with the zonotopic iterative approximation. Then, when the system is working in either healthy or faulty modes, the residual set characterizing these modes can be computed. The benefit of generating the residual in this way is to track the residual trajectories not only in steady state but also in transient state. Furthermore, in the case of having several types of faults, as long as the faulty and the healthy sets are separated, the proposed FDI approach will be able to work correctly.

In the case of IOA, the fault is detected by testing the consistency of the obtained residual based on the current behavior of the system and a fixed threshold (ideally 0). On the other hand, in the case of SIA, the residual invariant set in the healthy operation of the system is fixed and determined off-line. Then, the fault is detected by means of checking the inconsistency of the obtained residual of the current behaviour and the healthy residual set. Now, considering the zonotopic representation of an RPI set using Proposition 4.1 and through its iterative capability to generate the residual set, both FD principles can be combined. That is by using the same concept of SIA for FD test, that is based on checking if the obtained residual set belongs to healthy residual set of the system, instead of zero in the case of IOA. The healthy residual set can be generated at each time instant and compared with the current residual set. In this regard, not only the fault can be detected in both steady and transient states but also the fault can be isolated since the separated invariant sets representing different behaviors of the system can be obtained.

\subsection{Detectability and isolability conditions}

As mentioned before, the IOA can detect the fault in both transient and steady-state operation of a system. On the other hand, the FD test using the SIA can be applied only in steady state. But, in SIA, the invariant residual set that introduces the healthy operation of the system is computed off-line. In this regard, both healthy and faulty residual sets can be separated and considering this separation, the fault can be both detected and isolated. Furthermore, both on-line and off-line analysis can be used to generate the invariant set characterizing the residual, i.e.,

$r=\underbrace{y-\hat{y}}_{\text {on-line }}=\underbrace{C(x-\hat{x})+E_{v} v}_{\text {off-line }}$. 


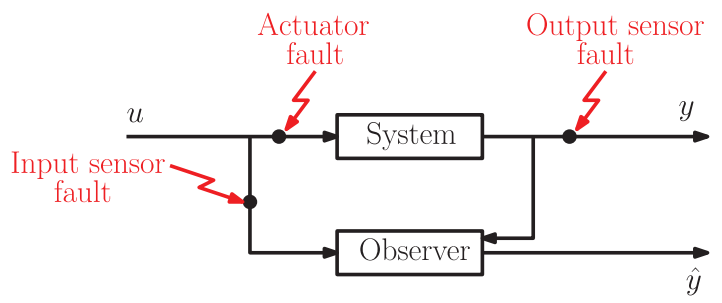

Fig. 1. Graphical interpretation of the different actuator and sensors faults.

The proposed method suggested in Proposition 4.1 leads to compute the residual in an on-line way. Therefore, after reaching the steady state, the computed residual set can be considered as an invariant set that combines the polytopic UB expression with the zonotopic iterative approximation. Then, if the system is working in healthy operation, this set introduces the healthy operation (that can be computed in both transient and steady states). Alternatively, in the case of occurrence of the fault, the residual trajectories can be bounded by another set that characterizes the faulty operation of the system. Then, the separation of the healthy and fault sets is associated to a fault occurrence. Moreover, in the case of having several faults such an observer is also be able to guarantee the isolability property. Given the classical Luenberger interval observer (5), it is possible to design one or several observers using Proposition 4.1 to satisfy the separation of the healthy and faulty sets to guarantee both detectability and isolability properties.

In this paper, different actuator and sensor faults will be considered. Including their effect, the dynamical model (4) can be rewritten as

$x_{+}=A x+B u+E_{\omega} \omega+F_{a} f_{a}$,

$y=C x+E_{v} v+F_{y} f_{y}$,

where vectors $f_{a} \in \mathbb{R}^{n_{u}}$ and $f_{y} \in \mathbb{R}^{n_{y}}$ denote the actuator and output sensor faults with their associated matrices $F_{a} \in \mathbb{R}^{n_{x} \times n_{u}}$ and $F_{y} \in \mathbb{R}^{n_{y} \times n_{y}}$, respectively. Furthermore, the other type of fault that is considered in this paper is known as input sensor fault which effect is considered on the input of the observer (5) as

$\hat{x}_{+}=A \hat{x}+B\left(u+F_{u} f_{u}\right)+L(y-\hat{y})$,

where $f_{u} \in \mathbb{R}^{n_{u}}$ represents the input sensor fault with its associated matrix $F_{u} \in \mathbb{R}^{n_{u} \times n_{u}}$. In addition, the considered faults can be classified into different categories depending on their locations as

- actuator faults, which affect the system inputs,

- sensor faults that affect the measurements of the inputs and outputs of the system.

Figure 1 shows the schematic graphical interpretation of the different actuator and sensors faults.

Assumption 4.1. The additive faults represented in Eqs. (23) and (24) are assumed to be bounded by a unit hypercube expressed as centered zonotopes, i.e., for all $k \geq 0, f_{\bullet} \in$ 
$\left\langle 0, I_{n_{f_{0}}}\right\rangle$, where the subscript - can be respectively assigned to $y, u$ or $a$ associated with the considered output sensor, input sensor and actuator faults, respectively.

Furthermore, the dynamics of state estimation error in Eq. (12) can be rewritten in faulty operation of the system as

$\tilde{x}_{+}=(A-L C) \tilde{x}+E_{d} d+F f$,

where

$F=\left[\begin{array}{lll}F_{a} & -L F_{y} & -B F_{u}\end{array}\right]$,

$f=\left[\begin{array}{lll}f_{a} & f_{y} & f_{u}\end{array}\right]^{T}$.

Remark 4.1. It is worth mentioning that, based on Assumption 4.1, only one fault can be considered at the same time in the observer structure ${ }^{2}$. Therefore, those elements of $F$ and $f$ that are related to the given fault are retained and the remainder elements are eliminated, e.g., in the case of an actuator fault, $F=\left[F_{a}\right]$ and $f=\left[f_{a}\right]$.

Considering $f \neq 0$, the effect of the uncertainty and fault should be considered when computing the zonotopic set bounding state estimation error that is defined in Proposition 4.2.

Proposition 4.2. Consider the dynamical model (23) and the observer (24), the decomposition of the center $c_{\tilde{x}}$ and the shape matrix $R_{\tilde{x}}$ of the zonotopic set bounding the state estimation error in Eq. (25) into the effects of the disturbance and fault can be recursively defined as

$\tilde{x}_{+} \in\left\langle c_{\tilde{x}_{d_{+}}}, R_{\tilde{x}_{d_{+}}}\right\rangle \oplus\left\langle c_{\tilde{x}_{f_{+}}}, R_{\tilde{x}_{f_{+}}}\right\rangle$,

with

$c_{\tilde{x}_{+}}=(A-L C) c_{\tilde{x}_{d}}$,

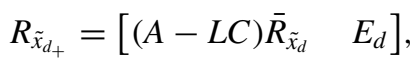

$c_{\tilde{x}_{f_{+}}}=(A-L C) c_{\tilde{x}_{f}}$,

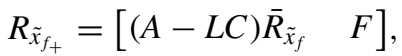

where the subscripts $d$ and $f$ denote the effects of uncertainties (i.e., state disturbance and measurement noise) and the fault, receptively.

Proof. Assume $\tilde{x} \in\left\langle c_{\tilde{x}_{d}}, R_{\tilde{x}_{d}}\right\rangle \oplus\left\langle c_{\tilde{x}_{f}}, R_{\tilde{x}_{f}}\right\rangle$ and consider Assumptions 2.1 and 4.1, the zonotopic form of the state estimation error in Eq. (17) can be expressed as

$x_{+} \in\left\langle(A-L C) c_{\tilde{x}_{d}},(A-L C) R_{\tilde{x}_{d}}\right\rangle \oplus\left\langle(A-L C) c_{\tilde{x}_{f}}, \quad(A-L C) R_{\tilde{x}_{f}}\right\rangle \oplus\left\langle 0, E_{d}\right\rangle \oplus\langle 0, F\rangle$.

Furthermore, consider that the superposition principle can be explicitly invoked in the linear setting. Therefore, using Definition 3, the center and the generator matrices in Eq. (29) can be reorganized as in Eq. (28). Thus, $\tilde{x}_{+} \in\left\langle c_{\tilde{x}_{d_{+}}}, R_{\tilde{x}_{d_{+}}}\right\rangle \oplus\left\langle c_{\tilde{x}_{f_{+}}}, R_{\tilde{x}_{f_{+}}}\right\rangle$.

\footnotetext{
${ }^{2}$ Further information on detection, isolation and estimation of multiple faults can be found in $[43,44]$.
} 
Consequently, the state estimation error can be projected into the residual space using Eq. (22). Thus, Proposition 4.2 allows to derive the residual set decomposing the effects of the disturbance and fault as

$c_{r_{d_{+}}}=C c_{\tilde{x}_{d_{+}}}$,

$R_{r_{d_{+}}}=\left[\begin{array}{ll}C R_{\tilde{x}_{d_{+}}} & E_{v}\end{array}\right]$

$c_{r_{f_{+}}}=C c_{\tilde{x}_{f_{+}}}$,

$R_{r_{f_{+}}}=\left[C R_{\tilde{x}_{f_{+}}}\right]$.

Furthermore, the effects of the uncertainty and fault on the residual set can be known as the residual sensitivity with respect to the uncertainty and fault. Therefore, this type of on-line observer can be used in both transient and steady states to guarantee the fault detectability and isolability in the case of satisfaction of conditions in Theorem 4.1 and 4.2.

Theorem 4.1 (Detectability condition). Consider Definition 5 and the decomposed form of the residual set in Eq. (30), the fault will be detected if

$s_{f_{\bullet l},}>s_{d_{u}}$,

$s_{f_{\bullet, u}}<s_{d_{l}}$,

with

$s_{f_{\bullet}, l}=\left(C_{i} c_{r_{\bullet}}-\left\|C_{i} R_{r_{\bullet}}\right\|_{1}\right)+\left(C_{i} c_{r_{d}}-\left\|C_{i} R_{r_{d}}\right\|_{1}\right)$,

$s_{f_{\bullet}, u}=\left(C_{i} c_{r_{f_{\bullet}}}+\left\|C_{i} R_{r_{\bullet}}\right\|_{1}\right)+\left(C_{i} c_{r_{d}}+\left\|C_{i} R_{r_{d}}\right\|_{1}\right)$,

$s_{d_{l}}=C_{i} c_{r_{d}}-\left\|C_{i} R_{r_{d}}\right\|_{1}$,

$s_{d_{u}}=C_{i} c_{r_{d}}+\left\|C_{i} R_{r_{d}}\right\|_{1}$,

where $i$ corresponds to the $i^{\text {th }}$ row of the vector $C$. Moreover, $s_{f_{l}}$ and $s_{f_{u}}$ are the minimum and the maximum values of the zonotope support strip in the faulty case, respectively. Furthermore, $s_{d_{l}}$ and $s_{d_{u}}$ are the minimum and the maximum values of the zonotope support strip in the healthy case, respectively.

Proof. Consider (30) in faultless scenario, i.e., $f_{\bullet}=0, r \in\left\langle c_{r_{d}}, R_{r_{d}}\right\rangle$. But, in the case of faulty operation of the system $r \notin\left\langle c_{r_{d}}, R_{r_{d}}\right\rangle$. Therefore, it can be written that

$\left\langle c_{r_{d}}, R_{r_{d}}\right\rangle \oplus\left\langle c_{r_{f_{\bullet}}}, R_{r_{f_{\bullet}}}\right\rangle \notin\left\langle c_{r_{d}}, R_{r_{d}}\right\rangle$.

Then, by computing the zonotope support strip using Definition 5 for the residual sets in both healthy and faulty operations of the system, (33) will be obtained if the inequality in Eq. (31) is satisfied.

Theorem 4.2 (Isolability condition). Consider the decomposed form of the residual set in Eq. (30) and Definition 5 to compute the zonotope support strip, a necessary condition that 
should be added to detectability condition in Theorem 4.1 in order to guarantee the isolation of a fault $f_{\bullet_{p}}$ from a fault $f_{\bullet_{q}}$ is

$s_{f_{\bullet, l_{p}}}>s_{f_{\bullet, u_{q}}}$,

$s_{f_{\bullet, u_{p}}}<s_{f_{\bullet, l_{q}}}$,

with

$s_{f_{\bullet, l_{p}}}=C_{i} c_{r_{f_{\bullet} p}}-\left\|C_{i} R_{r_{f_{\bullet}}}\right\|_{1}$,

$s_{f_{\bullet, u p}}=C_{i} c_{r_{f_{\bullet} p}}+\left\|C_{i} R_{r_{f_{\bullet}}}\right\|_{1}$,

$s_{f_{\bullet, l q}}=C_{i} c_{r_{f_{\bullet} q}}-\left\|C_{i} R_{r_{f_{\bullet} q}}\right\|_{1}$,

$s_{f_{\bullet}, u_{q}}=C_{i} c_{r_{\bullet_{\bullet} q}}+\left\|C_{i} R_{r_{f_{\bullet}}}\right\|_{1}$,

where $s_{f_{\bullet} l_{p}}$ and $s_{f_{\bullet, u_{p}}}$ are the minimum and the maximum values of the zonotope support strip in the case of occurrence of $f_{\bullet_{p}}$, respectively. Furthermore, $s_{f_{\bullet_{l_{q}}}}$ and $s_{f_{\boldsymbol{\bullet}_{u_{q}}}}$ are the minimum and the maximum values of the zonotope support strip in the case of occurrence of $f_{\bullet}$, respectively.

Proof. The proof follows in the same way than Theorem 4.1. Then, consider Eq. (30) and (31), it can be written that $f_{\bullet_{p}}$ is isolable from $f_{\bullet}$ if

$\left\langle c_{r_{f_{\bullet}}}, R_{r_{f_{\bullet p}}}\right\rangle \notin\left\langle c_{r_{f_{\bullet q}}}, R_{r_{f_{\bullet q}}}\right\rangle$.

Thus, Eq. (36) can be written using Definition 5 as Eq. (34) for the purpose of isolation of $f_{\bullet p}$ from $f_{\bullet_{q}}$.

Up to now, the paper has focused on detectability and isolability of presented approach during both transient and steady states operations of the system. It is mentioned that the detectability and isolability can be achieved in the case of satisfaction of the Theorems 4.1 and 4.2. Then, MDF and MIF can be characterized using the obtained conditions in the next section.

\subsection{Characterization of MDF and MIF}

To characterize the MDF and MIF based on the obtained conditions in Theorems 4.1 and 4.2, the input-output form of the measurement $y$ in Eq. (23) can be written as

$y=\mathscr{H}_{u}\left(q^{-1}\right) u+\mathscr{H}_{\omega}\left(q^{-1}\right) \omega+\mathscr{H}_{v}\left(q^{-1}\right) v+\mathscr{H}_{f_{a}}\left(q^{-1}\right) f_{a}+\mathscr{H}_{f_{y}}\left(q^{-1}\right) f_{y}$,

with

$\mathscr{H}_{u}\left(q^{-1}\right)=C \xi_{1}\left(q^{-1}\right) B$,

$\mathscr{H}_{\omega}\left(q^{-1}\right)=C \xi_{1}\left(q^{-1}\right) E_{\omega}$

$\mathscr{H}_{v}\left(q^{-1}\right)=E_{v}$,

$\mathscr{H}_{f_{a}}=C \xi_{1}\left(q^{-1}\right) F_{a}$ 
$\mathscr{H}_{f_{y}}=F_{y}$,

where $\xi_{1}\left(q^{-1}\right)=(q I-A)^{-1}$.

On the other hand, the input-output form of the output prediction $\hat{y}$ in Eq. (5b) can be expressed using the observer (24) as

$\hat{y}=\mathscr{T}_{u}\left(q^{-1}\right) u+\mathscr{T}_{y}\left(q^{-1}\right) y+\mathscr{T}_{f_{u}}\left(q^{-1}\right) f_{u}$,

with

$\mathscr{T}_{u}\left(q^{-1}\right)=C \xi_{2}\left(q^{-1}\right) B$

$\mathscr{T}_{y}\left(q^{-1}\right)=C \xi_{2}\left(q^{-1}\right) L$

$\mathscr{T}_{f_{u}}\left(q^{-1}\right)=C \xi_{2}\left(q^{-1}\right) B F_{u}$,

where $\xi_{2}\left(q^{-1}\right)=(q I-(A-L C))^{-1}$.

Then, the input-output form of the residual in Eq. (22) can be expressed using the shift operator $q^{-1}$ and considering Eqs. (37) and (39) as

$r=\mathscr{G}_{u}\left(q^{-1}\right) u+\mathscr{G}_{d}\left(q^{-1}\right) d+\mathscr{G}_{f}\left(q^{-1}\right) f$,

where the transfer function $\mathscr{G}_{u}$ denotes the effect of input on the residual that can be obtained as

$\mathscr{G}_{u}\left(q^{-1}\right)=\left(I-\mathscr{T}_{y}\left(q^{-1}\right)\right) \mathscr{H}_{u}\left(q^{-1}\right)-\mathscr{T}_{u}\left(q^{-1}\right)$,

and $\mathscr{G}_{d}=\left[\begin{array}{ll}\mathscr{G}_{\omega}\left(q^{-1}\right) & \mathscr{G}_{v}\left(q^{-1}\right)\end{array}\right]$ shows the effect of uncertainties on the residual and can be computed using

$\mathscr{G}_{\omega}\left(q^{-1}\right)=\left(I-\mathscr{T}_{y}\left(q^{-1}\right)\right) \mathscr{H}_{\omega}\left(q^{-1}\right)$,

$\mathscr{G}_{v}\left(q^{-1}\right)=\left(I-\mathscr{T}_{y}\left(q^{-1}\right)\right) \mathscr{H}_{v}\left(q^{-1}\right)$.

Furthermore, the effect of the fault is $\mathscr{G}_{f_{\bullet}}=\left[\begin{array}{lll}\mathscr{G}_{f_{a}}\left(q^{-1}\right) & \mathscr{G}_{f_{y}}\left(q^{-1}\right) & \mathscr{G}_{f_{u}}\left(q^{-1}\right)\end{array}\right]$ that can be computed using

$\mathscr{G}_{f_{a}}\left(q^{-1}\right)=\left(I-\mathscr{T}_{y}\left(q^{-1}\right)\right) \mathscr{H}_{f_{a}}\left(q^{-1}\right)$,

$\mathscr{G}_{f_{y}}\left(q^{-1}\right)=\left(I-\mathscr{T}_{y}\left(q^{-1}\right)\right) \mathscr{H}_{f_{y}}\left(q^{-1}\right)$

$\mathscr{G}_{f_{u}}\left(q^{-1}\right)=-\mathscr{T}_{f_{u}}\left(q^{-1}\right)$,

Then, considering input-output form of the residual in Eq. (41) and the detectability conditions in Theorem 4.1, the minimum magnitude of the fault that can be detected can be characterized following Theorem 4.3.

Theorem 4.3 (Minimum Detectable Fault). The MDF is characterized using conditions in (31), Proposition 4.2 and the decomposed form of the residual set in Eq. (30) as

$$
\overline{f_{\min _{\bullet_{j, \infty}}}^{\text {Det }}}=\max \overline{f_{\min _{\bullet_{j i, \infty}}}^{\text {Det }}}, \quad \overline{f_{\min _{\bullet_{j i, \infty}}}^{\text {Det }}}=+2 \frac{\left\|\mathscr{G}_{d_{i}}(1)\right\|_{1}}{\left\|\mathscr{G}_{\mathfrak{\bullet}_{i j}}(1)\right\|_{1}},
$$




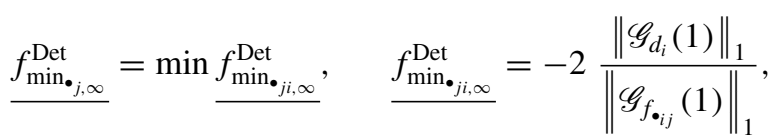

where the superscript Det refers to the detectable fault and the factor 2 appears because the worst-case scenario is considered, where the uncertainties have a maximum influence in the opposite direction compared to that of the fault occurrence. The indices $i$ and $j$ refer to the $i^{\text {th }}$ row and $j^{\text {th }}$ column of the transfers $\mathscr{G}_{d}$ and $\mathscr{G}_{f}$ (residual sensitivity with respect to the uncertainty and fault), respectively.

Proof. Based on Eq. (30), in the time domain and in steady state (limit as $k \rightarrow \infty), r_{\infty} \in$ $\left\langle c_{r_{d \infty}}, R_{r_{d \infty}}\right\rangle \oplus\left\langle c_{r_{f \infty}}, R_{r_{f \infty}}\right\rangle$. On the other hand, considering $f_{\text {min. }}^{\text {Det }} \in\left[f_{\min _{\bullet}}^{\text {Det }}, \overline{f_{\text {min. }}^{\text {Det }}}\right]$, it can be written that

$f_{\min .}^{\text {Det }} \in\left\langle c_{f_{\text {min }}^{\text {Det }}}, R_{f_{\text {min }}^{\text {Det }}}\right\rangle$,

where $c_{f_{\min }^{\text {Det }}}$ and $R_{f_{\min }^{\text {Det }}}$ show the center and the shape matrix of the MDF zonotope, respectively. Considering the worst-case scenario, the limit of the MDF can be understood as a maximum and minimum value of its zonotope support strip that can be computed using (46) and Definition 5. Therefore, the MDF is known as

$\overline{f_{\text {min }_{\bullet j}}^{\text {Det }}}=\max \overline{f_{\text {min }_{\bullet j}}^{\text {Det }}}=C_{i} c_{f_{\text {min }}^{\text {Det }}}+\left\|C_{i} R_{f_{\text {min }}^{\text {Det }}}\right\|_{1}$,

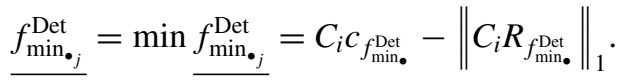

Then, consider $\omega \in\left\langle 0, I_{n_{\omega}}\right\rangle$ and $v \in\left\langle 0, I_{n_{v}}\right\rangle$, in the steady state for the faulty case, i.e., $f \cdot \neq 0$, it is satisfied

$r_{\infty} \in\left\langle\mathscr{G}_{u}(1) u_{\infty}, 0\right\rangle \oplus\left\langle 0, \mathscr{G}_{d}(1)\right\rangle \oplus\left\langle\mathscr{G}_{f_{\bullet}}(1) c_{f_{\text {min }}^{\text {Det }}}, \mathscr{G}_{f_{\bullet}}(1) R_{f_{\text {min }}^{\text {Det }}}\right\rangle$.

Moreover, the decomposed form of the center and the shape matrix of residual zonotope in steady state can be computed using Eq. (48) as

$c_{r_{\infty}}=\mathscr{G}_{u}(1) u_{\infty}$

$R_{r_{d \infty}}=\left[\mathscr{G}_{d}(1)\right]$

$c_{r_{f_{\bullet}, \infty}}=\mathscr{G}_{f_{\bullet}}(1) c_{f_{\text {min }}^{\text {Det }}}$,

$R_{r_{f_{\bullet} \infty}}=\left[\mathscr{G}_{f_{\bullet}}(1) R_{f_{\text {min }}^{\text {Det }}}\right]$,

where the subindex $r_{d_{\infty}}$ and $r_{f_{\infty}}$ show the residual sensitivity with respect to the effect of uncertainty and fault in steady state, respectively.

Now, consider Theorem 4.1 in the faulty operation of the system, i.e., satisfaction of the detectability conditions in Eq. (31), it can be stated that in steady state, the fault will be detected if

$s_{f_{\bullet, l, \infty}}>s_{d_{u, \infty}}$,

$s_{f_{\bullet, u, \infty}}<s_{d_{l, \infty}}$. 
Then, consider Eq. (49) and Definition 5, it can be written that

$s_{f_{\bullet}, l, \infty}=\left(C_{i} c_{r_{f_{\bullet}, \infty}}-\left\|C_{i} R_{r_{f_{\bullet}, \infty}}\right\|_{1}\right)+\left(C_{i} c_{r_{d_{\infty}}}-\left\|C_{i} R_{r_{d_{\infty}}}\right\|_{1}\right)$,

$s_{f_{\bullet}, u, \infty}=\left(C_{i} c_{r_{f_{\bullet}, \infty}}+\left\|C_{i} R_{r_{f_{\bullet}, \infty}}\right\|_{1}\right)+\left(C_{i} c_{r_{d_{\infty}}}+\left\|C_{i} R_{r_{d_{\infty}}}\right\|_{1}\right)$,

$s_{d_{l, \infty}}=C_{i} c_{r_{d \infty}}-\left\|C_{i} R_{r_{d \infty}}\right\|_{1}$,

$s_{d_{u, \infty}}=C_{i} c_{r_{d \infty}}+\left\|C_{i} R_{r_{d \infty}}\right\|_{1}$.

Next, by considering the worst-case scenario, i.e., the residual is considered with the extreme value (it is located at either the lower or the upper bound of the zonotope support strip of the considered threshold), it follows that the fault can be detected if

$\left(C_{i} c_{r_{f_{\bullet}, \infty}}-\left\|C_{i} R_{r_{f_{\bullet}, \infty}}\right\|_{1}\right)>+2\left\|C_{i} R_{r_{d_{\infty}}}\right\|_{1}$,

$\left(C_{i} c_{r_{f_{\bullet}, \infty}}+\left\|C_{i} R_{r_{f_{\bullet}, \infty}}\right\|_{1}\right)<-2\left\|C_{i} R_{r_{d_{\infty}}}\right\|_{1}$.

Finally, the MDF can be characterized considering Eq. (47) and by substitution of residual sensitivity (49) and Eq. (51) in Eq. (52), which results in Eq. (45).

The condition in Theorem 4.1 is sufficient only for detecting the fault and the MDF, which is characterized in Theorem 4.3. Moreover, the fault can be isolated if the intersection between the residual sets (computed based on different type of faults) is empty. Therefore, the condition in Theorem 4.2 should also be satisfied together with condition (31) in order to guarantee both detection and isolation of the fault.

Furthermore, using residual in Eq. (30) and condition in Theorem 4.2, the minimum magnitude of the fault that can guarantee both detection and isolation is characterized following Theorem 4.4.

Theorem 4.4 (Minimum Isolable Fault). The MIF of a fault $f_{\bullet_{p}}$ from a fault $f_{\bullet_{q}}$ is characterized using the conditions in Theorems 4.1 and 4.2, and also, considering the decomposed form of the residual set in (30) as

$\overline{f_{\min _{\bullet_{j}, \infty}}^{\text {Iso }}}=\max \overline{f_{\min _{\bullet_{p_{j i}, \infty}}}^{\text {Iso }}}$,

$$
\overline{f_{\min _{\bullet_{j i}, \infty}}^{\text {Iso }}}=+2 \frac{\left\|\mathscr{G}_{d_{i}}(1)\right\|_{1}}{\left\|\mathscr{G}_{f_{\bullet_{i j}}}(1)\right\|_{1}}+\frac{\left\|\mathscr{G}_{f_{\bullet_{q_{i}}}}(1)\right\|_{1}}{\left\|\mathscr{G}_{f_{\bullet_{p_{i j}}}}(1)\right\|_{1}},
$$

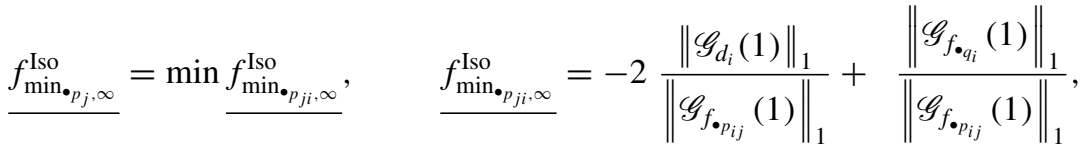

where the superscript Iso refers to the isolable fault and the factor 2 appears because the worst-case scenario is considered considering the effect of uncertainties $d$ and fault $f_{\bullet_{q}}$ that have a maximum influence in the opposite direction compared to that of the fault occurrence $f_{\bullet}$.

Proof. The proof follows a similar procedure than the one used in Theorem 4.3. In this regard, based on Eq. (30), it can be written in the time domain and in steady state (limit as $k \rightarrow \infty$ ) that $r_{\infty} \in\left\langle c_{r_{d_{\infty}}}, R_{r_{d_{\infty}}}\right\rangle \oplus\left\langle c_{r_{f_{\bullet}, \infty}}, R_{r_{f_{\bullet}, \infty}}\right\rangle$ in the case of occurrence of $f_{\bullet_{p}}$. Moreover, $r_{\infty} \in$ 
$\left\langle c_{r_{d_{\infty}}}, R_{r_{d_{\infty}}}\right\rangle \oplus\left\langle c_{r_{f_{\bullet_{q}, \infty}}}, R_{r_{f_{\bullet_{q}, \infty}}}\right\rangle$ in the case of occurrence of $f_{\bullet_{q}}$. On the other hand, considering $f_{\min _{\bullet p}}^{\text {Iso }} \in\left[\overline{f_{\min _{\bullet p}}^{\text {Iso }}}, \overline{f_{\text {min }_{\bullet p}}^{\text {Iso }}}\right]$, it can be written that

$f_{\text {min }_{\bullet p}}^{\text {Iso }} \in\left\langle c_{f_{\text {min }_{\bullet p}}^{\text {Iso }}}, R_{f_{\text {min } \bullet_{p}}^{\text {Iso }}}\right\rangle$,

$f_{\min _{\bullet} q}^{\text {Iso }} \in\left\langle c_{f_{\text {min }_{\bullet}}^{\text {Iso }}}, R_{f_{\text {min }_{\bullet}}^{\text {Iso }}}\right\rangle$,

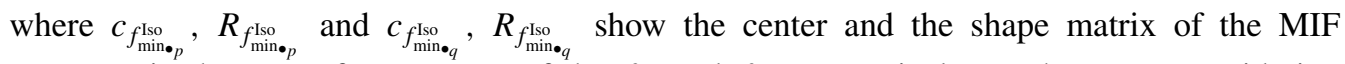
zonotope in the case of occurrence of the $f_{\bullet_{p}}$ and $f_{\bullet_{q}}$, respectively. Furthermore, considering the isolability condition in Theorem 4.2 , in the case of occurrence of $f_{\bullet p}$, i.e., $f_{\bullet p} \neq 0$, and when $k \rightarrow \infty$, it can be derived that

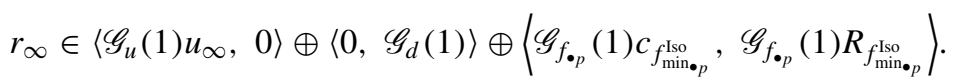

On the other hand, for the case of occurrence of $f_{\bullet_{q}}$, it is satisfied

$r_{\infty} \in\left\langle\mathscr{G}_{u}(1) u_{\infty}, 0\right\rangle \oplus\left\langle 0, \mathscr{G}_{d}(1)\right\rangle \oplus\left\langle\mathscr{G}_{f_{\bullet_{q}}}(1) c_{f_{\text {min }_{\bullet q}}^{\text {Iso }}}, \mathscr{G}_{f_{\bullet_{q}}}(1) R_{f_{\text {min }}^{\text {Iso }}}\right\rangle$

Now, the residual sensitivity with respect to the effect of uncertainties in steady state can be obtained as in Eq. (49a) and (49b). Furthermore, the residual sensitivity with respect to the effect of faults $f_{\bullet_{p}}$ and $f_{\bullet_{q}}$ in steady state can be computed as

$c_{r_{f_{\bullet}, \infty}}=\mathscr{G}_{f_{\bullet_{p}}}(1) c_{f_{\text {min }_{\bullet p}}^{\mathrm{Iso}}}$,

$R_{r_{f_{\bullet}, \infty}}=\left[\mathscr{G}_{f_{\bullet p}}(1) R_{f_{\text {min } n_{\bullet}}^{\text {Iso }}}\right]$,

$c_{r_{f_{\bullet}, \infty}}=\mathscr{G}_{f_{\bullet_{q}}}(1) c_{f_{\text {min }_{\bullet} \text { Iso }}}$,

$R_{r_{f_{\bullet}, \infty}}=\left[\mathscr{G}_{f_{\bullet_{q}}}(1) R_{f_{\text {min }_{\bullet} q}^{\text {Iso }}}\right]$.

Besides, the isolability condition in Eq. (34) can be rewritten during steady state as

$s_{f_{\bullet, l_{p}, \infty}}>s_{f_{\bullet, u_{q}, \infty}}$,

$s_{f_{\bullet, u_{p}, \infty}}<s_{f_{\bullet, l_{q}, \infty}}$,

where

$s_{f_{\bullet}, l_{p}, \infty}=\left(C_{i} c_{r_{f_{\bullet}, \infty}}-\left\|C_{i} R_{r_{f_{\bullet}, \infty}}\right\|_{1}\right)+\left(C_{i} c_{r_{d_{\infty}}}-\left\|C_{i} R_{r_{d_{\infty}}}\right\|_{1}\right)$,

$s_{f_{\bullet, u_{p}, \infty}}=\left(C_{i} c_{r_{f_{\bullet}, \infty}}+\left\|C_{i} R_{r_{f_{\bullet}, \infty}}\right\|_{1}\right)+\left(C_{i} c_{r_{d_{\infty}}}-\left\|C_{i} R_{r_{d_{\infty}}}\right\|_{1}\right)$,

$s_{f_{\bullet}, l_{q}, \infty}=\left(C_{i} c_{r_{f_{\bullet}, \infty}}-\left\|C_{i} R_{r_{f_{\bullet}, \infty}}\right\|_{1}\right)+\left(C_{i} c_{r_{d \infty}}-\left\|C_{i} R_{r_{d \infty}}\right\|_{1}\right)$,

$s_{f_{\bullet}, u_{q}, \infty}=\left(C_{i} c_{r_{f_{\bullet}, \infty}}+\left\|C_{i} R_{r_{f_{\bullet}, \infty}}\right\|_{1}\right)+\left(C_{i} c_{r_{d_{\infty}}}-\left\|C_{i} R_{r_{d_{\infty}}}\right\|_{1}\right)$.

Then, by considering the worst-case scenario for both uncertainties and the considered fault (here $f_{\bullet_{q}}$ ) with respect to the other faults (here $f_{\bullet_{p}}$ ), the isolability condition can be rewritten as

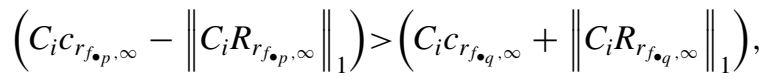


$\left(C_{i} c_{r_{f_{0}, \infty}}+\left\|C_{i} R_{r_{f_{0} \infty}}\right\|_{1}\right)<\left(C_{i} c_{r_{f_{f_{q}}, \infty}}-\left\|C_{i} R_{r_{f_{\sigma_{q}}, \infty}}\right\|_{1}\right)$.

Finally, the MIF can be characterized considering Eq. (54) and by substitution of the residual sensitivity with respect to disturbances (49a), (49b) and the residual sensitivity with respect to faults (57) in Eq. (60) results in Eq. (53a).

The minimum magnitude of the fault that establishes if a fault is detectable and isolable according to Theorems 4.3 and 4.4. Therefore, it can be written that in the case of satisfaction of both conditions in Eqs. (31) and (34), the fault can be detected and isolated. Furthermore, another possible manner to guarantee both detection and isolation at the same time can be achieved by considering Eq. (60). This condition shows that the isolation of the fault depends on the direction of vector $C$. Thus, in the case of having the occurrence of the faults in different directions, the fault can be isolated with the same magnitude of the MDF. In this regard, further analysis of the approaches will be discussed in Section 5 based on a case study.

\section{Case study}

\subsection{System description}

The proposed FDI scheme will be tested using a two-tank system based on the well-known benchmark proposed in [45]. A schematic of the system can be seen in Fig. 2.

The input of the two-tank system is the pump flow rate that is determined when applying voltage $v$ of the pump. Therefore, the action of the pump is to pour the tanks by extracting the water from the basin. Moreover, Tank 1 is placed below Tank 2. Furthermore, the outputs of the process are the water levels in both upper and lower tanks that are obtained as voltages from the measurement devices.

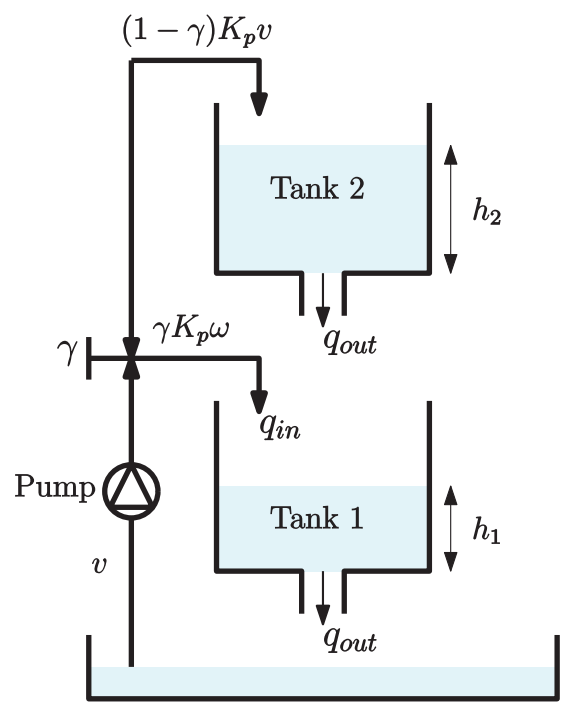

Fig. 2. Schematic diagram of the Two-tanks system. 
Additionally, Tank 1 is being affected by an additional disturbance $\omega$ that is generated by the uncertain position of the valve $\gamma$ that can vary between 0 and 1 based on experimental apparatus, i.e., the position of the valve is the ratio modeling how the output of the pump is divided between upper and lower tanks. Thus, the water flow to each tank is controlled by the position of the valve considered as $\gamma \in(0,1)$. Furthermore, both upper and lower tanks are made from Plexiglas tubes with the height of $20 \mathrm{~cm}$ that are connected by the pipe with a diameter of $6 \mathrm{~cm}$.

Since all the mathematical developments in previous sections were obtained based on the consideration of a linear dynamic system, the linearized model of the considered two-tank system is used to illustrate the effectiveness of the characterized formulations. According to [45], the two-tank model is linearized around a working point. The considering operating point is denoted using the superscript $*$ and selected as

- $h_{1}^{*}=12.4 \mathrm{~cm}$,

- $h_{2}^{*}=1.8 \mathrm{~cm}$,

- $v^{*}=3.00 \mathrm{~V}$,

with the following parameter values:

- $K_{p}=3.35 \mathrm{~cm}^{3} / \mathrm{Vs}$

- $\gamma=0.60$.

Hence, the following linearized model can be written by introducing the variables $\tilde{h}_{i}=$ $h_{i}-h_{i}^{*}$ and $\tilde{v}=v-v^{*}$, where $h_{i}$ is the level of the water in Tank $i$, with $i=1.2$ and $v$ is the velocity of the water flow through the pump:

$\dot{\tilde{h}}_{t}=\left[\begin{array}{cc}-\frac{1}{T_{1}} & \frac{A_{2}}{A_{1} T_{2}} \\ 0 & -\frac{1}{T_{2}}\end{array}\right] \tilde{h}_{t}+\left[\begin{array}{cc}\frac{\gamma K_{p}}{A_{1}} & 0 \\ 0 & \frac{(1-\gamma) K_{p}}{A_{2}}\end{array}\right] \tilde{v}_{t}$,

$y_{t}=\left[\begin{array}{ll}K_{c} & 0\end{array}\right] \tilde{h}_{t}$,

where $K_{c}$ is measured laboratory parameter. Moreover, $T_{i}=\frac{A_{i}}{a_{i}} \sqrt{\frac{2 h_{i}^{*}}{g}}$ with $i=1,2$.

According to [45], the parameters of the model in Eq. (61) are $A_{1}=A_{2}=28 \mathrm{~cm}^{2}, a_{1}=$ $a_{2}=0.071 \mathrm{~cm}^{2}, K_{c}=0.50 \mathrm{~V} / \mathrm{cm}$ and $g=981 \mathrm{~cm} / \mathrm{s}^{2}$. Therefore, $T_{1}=62.7034 \mathrm{~s}$ and $T_{2}=$ $23.8900 \mathrm{~s}$.

Using the Euler discretization with a sampling time of $1 \mathrm{~s}$, the linearized model of this system can be written in the state-space form as

$\tilde{h}_{+}=A \tilde{h}+B \tilde{v}+E_{\omega} \omega$

$y=C \tilde{h}+E_{v} v$,

where $A=\left[\begin{array}{cc}0.9842 & 0.0407 \\ 0 & 0.9590\end{array}\right], B=\left[\begin{array}{l}0.0007 \\ 0.0352\end{array}\right]$ and $C=\left[\begin{array}{cc}0.5 & 0 \\ 0 & 0.5\end{array}\right]$. Furthermore, taking into account the state disturbance and the measurement noise, $E_{\omega}$ and $E_{v}$ are simulated in 

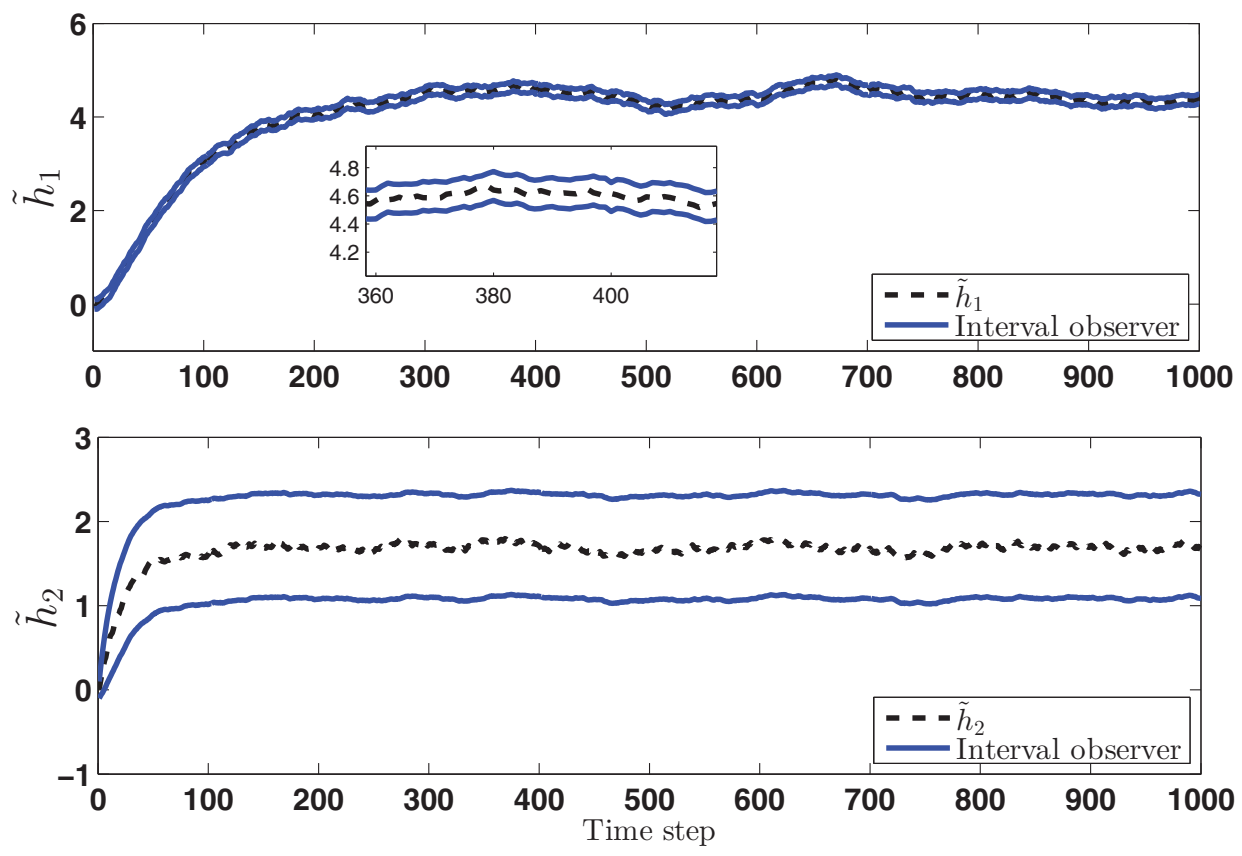

Fig. 3. State estimation in healthy operation of the system.

Eq. (62) with

$E_{\omega}=\left[\begin{array}{cc}0.05 & 0 \\ 0 & 0.05\end{array}\right], \quad E_{v}=\left[\begin{array}{cc}0.01 & 0 \\ 0 & 0.01\end{array}\right]$.

As it can observed in Eq. (63), $E_{\omega}$ is used to define a disturbance influencing all the states and the measurement noise affecting both outputs is modeled through $E_{v}$.

Based on Eq. (62), the output $y$ is the voltage obtained from the level sensor. Hence, considering the physical features of the two-tank system, the range of the measured output is $[0,10] \mathrm{V}$ since the height of the each tank is from $0 \mathrm{~cm}$ up to $20 \mathrm{~cm}$ and $K_{c}=0.5 \mathrm{~V} / \mathrm{cm}$. Moreover, based on linear model in Eq. (61), the incremental value of the measured output around the working point is in the range of $4 \mathrm{~V}$ to $8 \mathrm{~V}$ (or $8 \mathrm{~cm}$ to $16 \mathrm{~cm}$ ).

\subsection{Performing FDI}

\subsubsection{Healthy operation of the system}

The first step of the proposed FDI approach is to obtain the state estimation. In this regard, the additive uncertainties ( $\omega$ and $v$ ) are assumed unknown but bounded based on the zonotopic definition of a set during the simulations as in (3). Fig. 3 shows the projection of the computed state-bounding zonotope into the state-space when the system is working in its healthy mode.

As discussed in Sections (3.1) and (3.2), there are two different approaches for bounding the effect of uncertainty in the residual. On the one hand, the on-line IOA that is able to generate the residual set in both transient and steady states. On the other hand, the SIA that is an off-line procedure to compute the residual set in steady state (see (22)). Fig. 4 


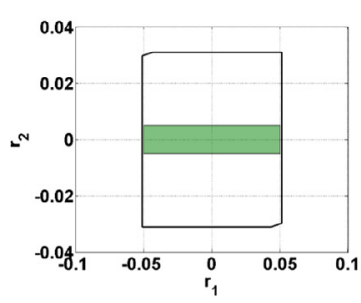

(a) $\mathrm{k}=1$.

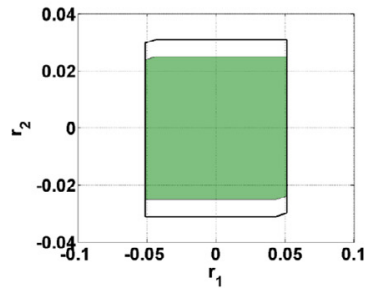

(d) $\mathrm{k}=30$.

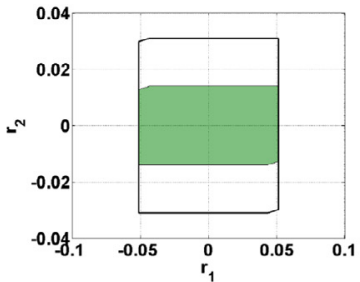

(b) $\mathrm{k}=10$.

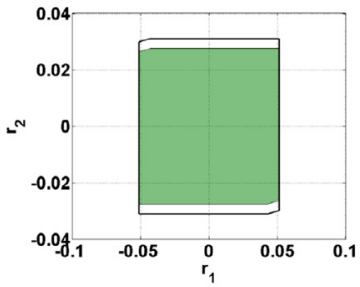

(e) $\mathrm{k}=40$.

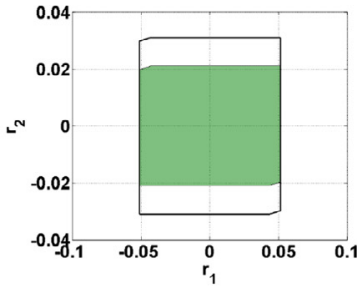

(c) $\mathrm{k}=20$.

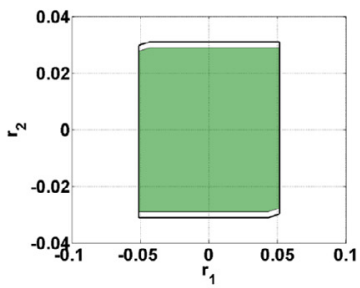

(f) $\mathrm{k}=50$.

Fig. 4. On-line propagation of residual set using zonotopic IOA during transient state and healthy operation of the system.

presents the residual set based on on-line IOA that is obtained from the transient operation of the healthy system. The obtained residual zonotopes at time instants $k=1, k=10, k=20$, $k=30, k=40$ and $k=50$ are shown in Figure 4 for the healthy functioning of the system in Eq. (62). From the results in Fig. 4, it can be seen that the residual generated using the proposed on-line zonotopic observer (the green zonotopes) ultimately converges to the one that is represented by the black solid line.

Based on the system description, outputs of the considered two-tank system are the water level in both upper and lower tanks. Therefore, the residual zonotopic set can be generated as a plane zonotope (2D zonotope) at each time step, where $r_{1}$ and $r_{2}$ denote the difference between the predicted values of the $\tilde{h}_{1}$ and $\tilde{h}_{2}$ with their real measured values given by the sensor, respectively.

From the results in Fig. 4, it can be observed that the residual generated by an on-line zonotopic observer is also converging to the one that is shown in Fig. 5, which can be considered as an RPI set for the residual. Furthermore, Fig. 5 shows the obtained residual set from the SIA based on (14).

What is interesting in Fig. 5 is related to the comparison of the residual zonotopes obtained based the off-line and on-line approaches. A comparison of the two results reveals that no significant differences were found between the size of the residual zonotopes in steady state. Therefore, the obtained RPI set for the residual based the proposed on-line zonotopic IOA is confirmed by the use of SIA. Furthermore, it is true that the difference between the computed RPI set is not significant but the size of the RPI set that is computed on-line is a bit tighter than the one computed off-line. Thus, it can be considered that the off-line SIA is more conservative than the on-line IOA since the RPI set is computed off-line. This result may be explained by the fact that the iterative propagation of the uncertainties in zonotopic IOA is more accurate than the off-line computation procedure. Furthermore, the mathematical burden is increased using the on-line approach but it allows obtaining a more accurate result. 


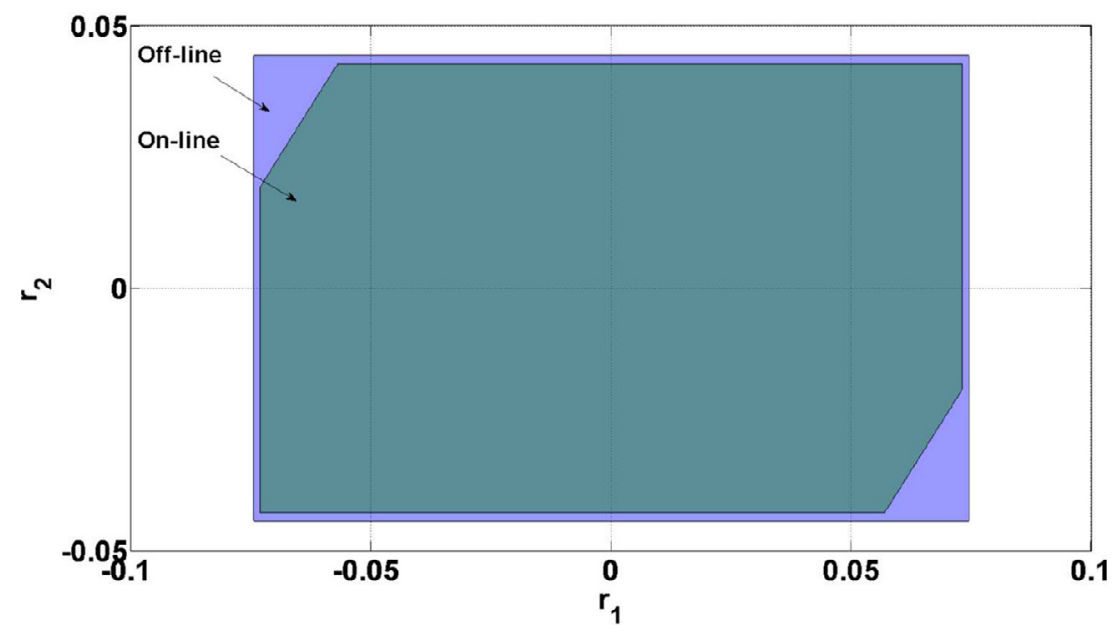

Fig. 5. Comparison of the residual set using on-line and off-line approaches in steady state.

This section presents the analysis of the system in healthy operation. The next step is to test the proposed so-called on-line IOA during the faulty case.

\subsubsection{Faulty operation of the system}

Following Section 4, the MDF can be computed using Theorem 4.3 for different types of actuator and sensor faults. The effect of faults on the state and the measurements are modeled through the components of matrix $F$ in Eq. (26). Furthermore, in order to analyze the effect of different type of faults on the system, actuator and sensor faults are simulated, separately.

In the first simulation, the actuator fault is considered. In this regard, the position of the valve is controlled by using the electrical actuator. As it is explained in the description of the case study, the position of the valve during the experiment is related to the flow parameter $\gamma$ in the range between $[0,1]$. Thus, the flow to the lower and upper tanks is influenced by the valve position through $\gamma K_{p} \omega$ and $(1-\gamma) K_{p} v$, respectively. To simulate the single actuator fault, faulty dynamical model (23) is considered as

$x_{+}=A x+B u+E_{\omega} \omega+F_{a} f_{a}$,

$y=C x+E_{v} v$.

From (64), it can be observed that system is affected by the actuator fault through matrix $F_{a}$ and the vector $f_{a}$, i.e.,

$F_{a}=10 B, \quad f_{a}=\left[f_{a}\right]$,

where matrix $F_{a}$ is selected to simulate the actuator fault. Moreover, $f_{a}$ denotes the direction of the fault effect on the actuator. Then, considering all the details mentioned regarding to the system simulation and simulation of the actuator fault, Theorem 4.3 can be used in order to compute the MDF. Therefore, the minimum magnitude of the actuator fault that can be detected is obtained using Eq. (45). Thus, the MDF in the case of actuator fault is computed during steady-state operation of the system as

$f_{\min _{a}}^{\text {Det }}= \pm 0.3310$. 

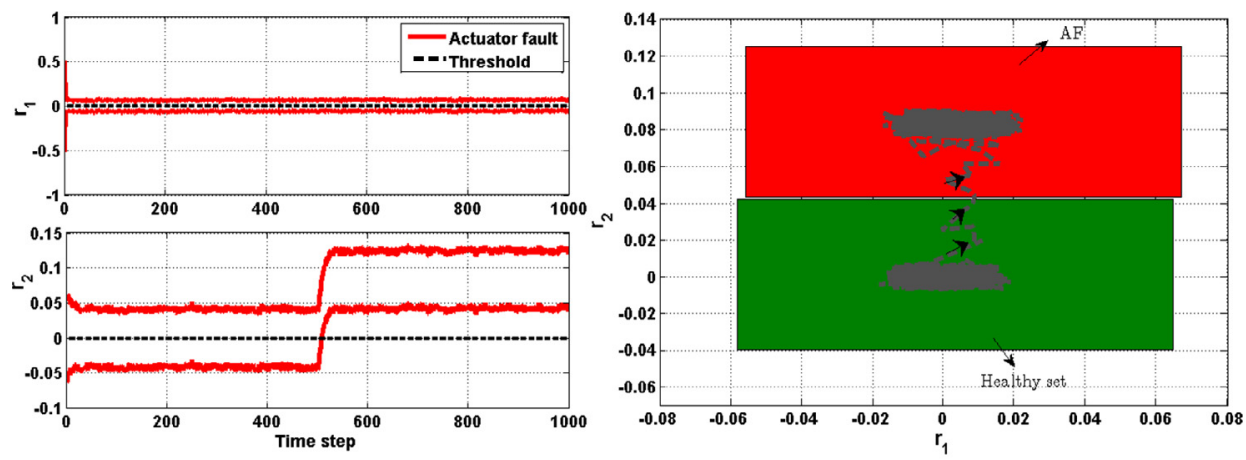

(a) $f_{a}=0.3330$ at $k=500$.
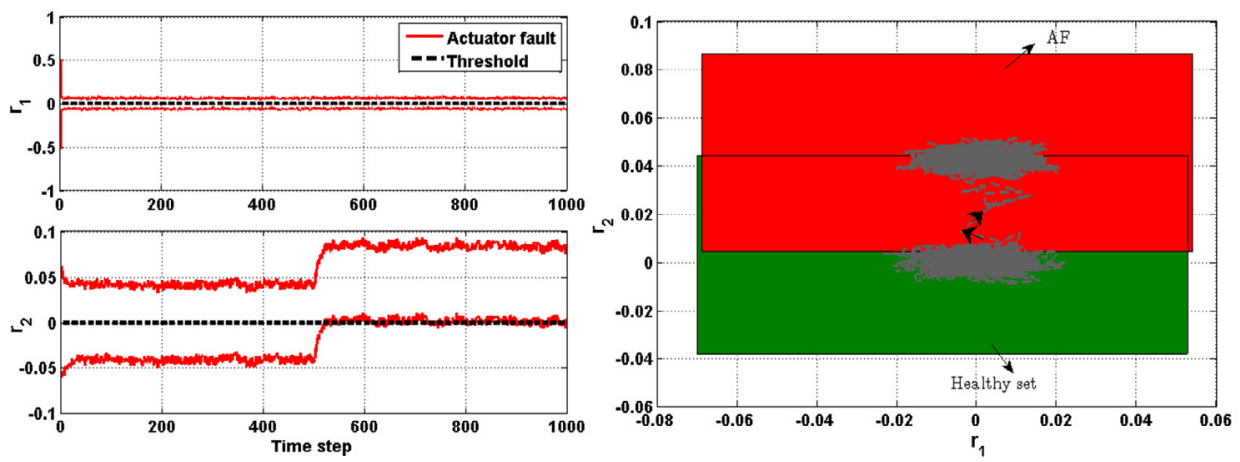

(b) $f_{a}=0.3280$ at $k=500$.

Fig. 6. FD results in the case of occurrence of the actuator fault during steady state, i.e., $k=500$.

To test the obtained magnitudes, the occurrence of the fault is simulated at $k=500$, which simulates the fault in steady state. Furthermore, based on the description of the case study, the flow parameter is considered as $\gamma=0.6$.

In this regard, the two following scenarios for a single step additive actuator fault are considered in steady state:

- scenario (i): $f_{a}=0.3330$, i.e, slightly bigger than the magnitude $f_{\min _{a}}^{\text {Det }}= \pm 0.3310$,

- scenario (ii): $f_{a}=0.3280$, i.e, slightly smaller than the magnitude $f_{\min _{a}}^{\text {Det }}= \pm 0.3310$.

Fig. 6 shows the FD test results for both scenarios. As it can be seen in Fig. 6, the residual sets obtained in healthy and faulty operation are separated in the case of fault occurrence considering the first scenario. Therefore, the fault can be detected. But, considering the second scenario, the overlap between the healthy and faulty residual sets means the fault with the considered magnitudes is not detectable.

Further analysis is carried out for the case of actuator fault at $k=500$ by projecting the faulty residual set into each residual space as can be seen on the left side of the Fig. 6. It can be observed that the threshold, i.e., ideally in classical IOA is considered zero, is out of the area between the upper and lower bounds of the residual set in Fig. 6a. Then, the existence of the fault will be proved. On the other hand, the threshold is between the area of 
the maximum and minimum bounds of the residual set in Fig. $6 \mathrm{~b}$, where the second scenario is considered for the fault magnitude. Therefore, the fault with the magnitude $f_{a}<0.3310$ is not detectable. Hence, Fig. 6 confirms through simulations the obtained results in (66). This illustrates that proposed on-line zonotopic IOA is well suited to the fault detectability of the classical IOA.

Remark 5.1. The healthy zonotopic set that is shown in Figs. 4 and 5 is obtained without considering the reduction operator $\downarrow_{q}$ since the computational burden is not to much for this case study. But, in the faulty operation of the system since the computational burden is increased, the reduction operator $\downarrow_{q}$ is used to fix the dimension of the generator matrices with the maximum value. Due to this reason, the healthy residual set that is shown in Figs. 5 and 6 are not exactly the same.

Moreover, the MDF analysis is done considering the output sensor fault. In this regards, the faulty dynamical model (23) is considered as

$x_{+}=A x+B u+E_{\omega} \omega$,

$y=C x+E_{v} v+F_{y} f_{y}$.

As mentioned in the description of the case study, the outputs of the system are the water levels in Tanks 1 and 2 that can be measured using the measurement devices as voltages. Based on the physical features, the height of each tank is $20 \mathrm{~cm}$. Then, each output of the system is between $[010] \mathrm{V}$ since $K_{c}=0.50 \mathrm{~V} / \mathrm{cm}$. To simulate the output sensor fault, the terms $F_{y}$ and $f_{y}$ in Eq. (67) is considered in the simulation as

$F_{y}=\left[\begin{array}{cc}10 & 0 \\ 0 & 10\end{array}\right], \quad f_{y}=\left[\begin{array}{l}f_{y_{1}} \\ f_{y_{2}}\end{array}\right]$,

where the matrix $F_{y}$ is defined with the whole range of the measurement. Moreover, $f_{y_{1}}$ and $f_{y_{2}}$ present the influence of the fault on each output. Then, the minimum magnitude of the sensor fault that can be detected can be computed based on Theorem 4.3. Using Eq. (45), the minimum magnitude of the output sensor fault that can be detected is computed during steady-state operation of the system as

$f_{\text {min }_{y}}^{\text {Det }}=\left[\begin{array}{l} \pm 0.2575 \mathrm{~V} \\ \pm 0.0082 \mathrm{~V}\end{array}\right]$

As further analysis, the occurrence of the output sensor fault is separately simulated at $k=500$, in order to test the obtained magnitude during steady state. Furthermore, based on the description of the case study, the operating points that are considered for the water levels of the Tanks 1 and 2 are around $12.4 \mathrm{~cm}$ (or $6.2 \mathrm{~V}$ ) and 1.8 (or $0.9 \mathrm{~V}$ ), respectively. Then, the FD test is done considering the following scenarios for a single step additive output sensor fault are considered during steady state:

- slightly bigger than the magnitude $f_{\min _{y}}^{\text {Det }}=\left[\begin{array}{l} \pm 0.2575 \mathrm{~V} \\ \pm 0.0082 \mathrm{~V}\end{array}\right]$,

- slightly smaller than the magnitude $f_{\min _{y}}^{\text {Det }}=\left[\begin{array}{l} \pm 0.2575 \mathrm{~V} \\ \pm 0.0082 \mathrm{~V}\end{array}\right]$.

Following the explained scenarios, there are four different magnitudes to be tested on each sensor as 

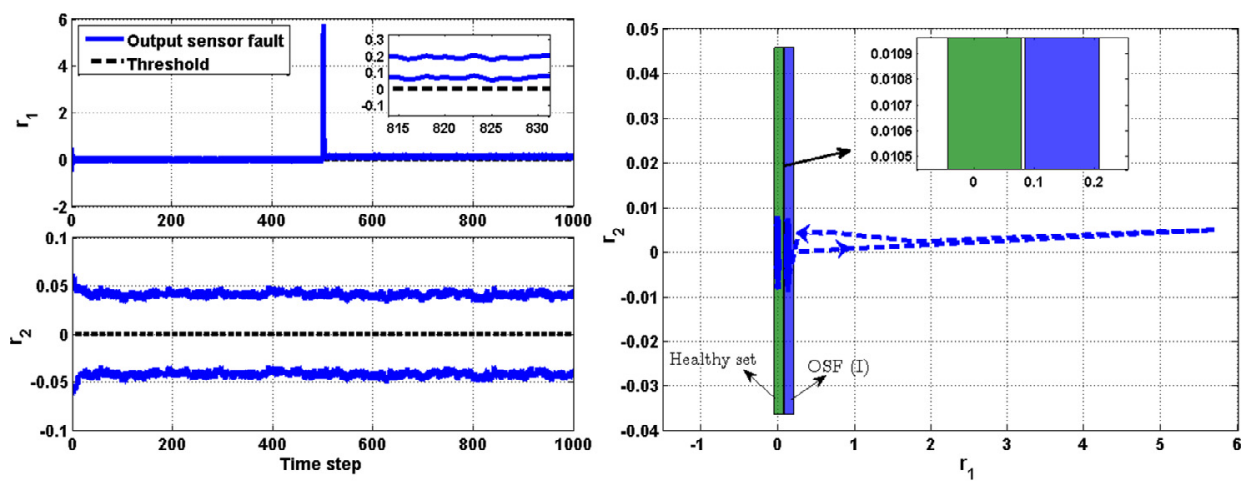

(a) $f_{y_{1}}=0.2580 \mathrm{~V}$ at $k=500$.
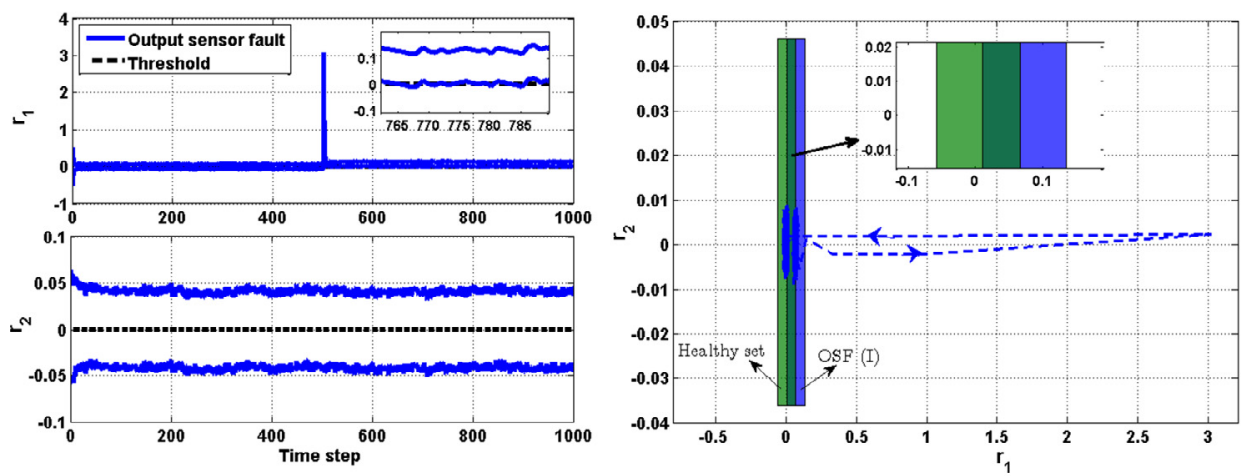

(b) $f_{y_{1}}=0.2570 \mathrm{~V}$ at $k=500$.

Fig. 7. FD results in the case of occurrence of the sensor fault $\left(f_{y_{1}}\right)$ during steady state, i.e., $k=500$.

$\circ$ scenario (i): $f_{y_{1}}=0.2580 \mathrm{~V}$, i.e, slightly bigger than the magnitude $f_{\min _{y_{1}}}^{\text {Det }}= \pm 0.2575 \mathrm{~V}$, $\circ$ scenario (ii): $f_{y_{1}}=0.2570 \mathrm{~V}$, i.e, slightly smaller than the magnitude $f_{\min _{y_{1}}}^{\text {Det }}= \pm 0.2575 \mathrm{~V}$, $\circ$ scenario (iii): $f_{y_{2}}=0.0084 \mathrm{~V}$, slightly bigger than the magnitude $f_{\min _{y_{2}}}^{\text {Det }}= \pm 0.0082 \mathrm{~V}$, $\circ$ scenario (iv): $f_{y_{2}}=0.0080 \mathrm{~V}$, slightly smaller than the magnitude $f_{\min _{y_{2}}}^{\text {Det }}= \pm 0.0082 \mathrm{~V}$.

It is worth mentioning that subscripts 1 and 2 denote the effect of the fault on the sensor that is measuring the water level of Tanks 1 and 2, respectively. The results from the implementation of the scenarios are reported in Figs. 7 and 8.

Looking at Figs. 7 and 8, it can be observed that separation of the healthy and faulty residual sets is obtained when slightly bigger faults than the magnitudes obtained in Eq. (69) are considered and the existence of the fault can be detected by means of the obtained separations. Considering the second scenario, which corresponds to a fault slightly smaller than the obtained magnitudes in Eq. (69) is considered, the fault can not be detected since the healthy and faulty residual sets overlap. Furthermore, it can be seen that the threshold, i.e., zero, is out of the area between the upper and lower bounds of the residual set when implementing the first scenario of the two cases. Then, this is an indication of the occurrence of the fault. Moreover, threshold is inside of the area between the upper and lower bounds of the faulty residual set when implementing the second scenario of the two cases and it can be considered 

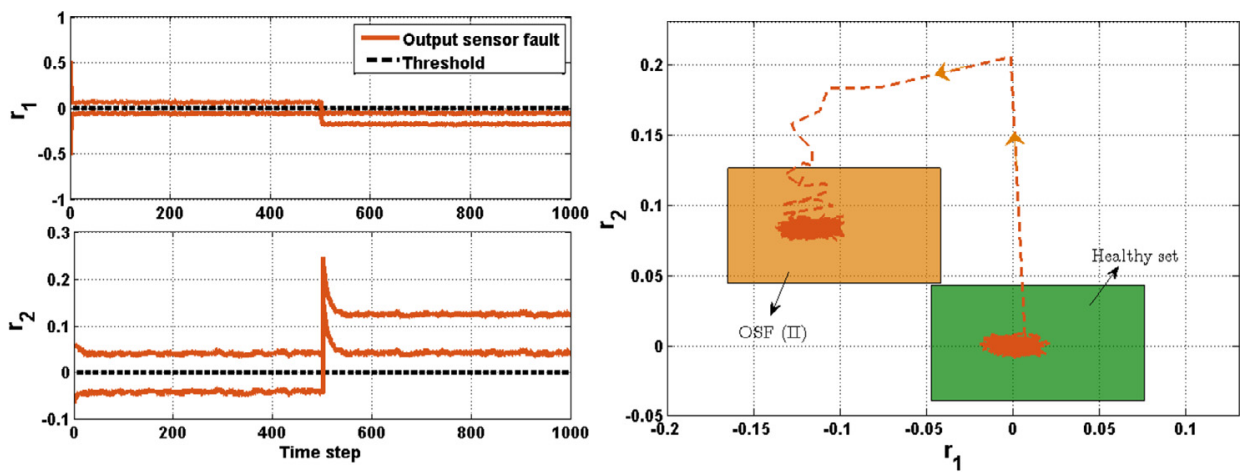

(a) $f_{y_{2}}=0.0084 \mathrm{~V}$ at $k=500$
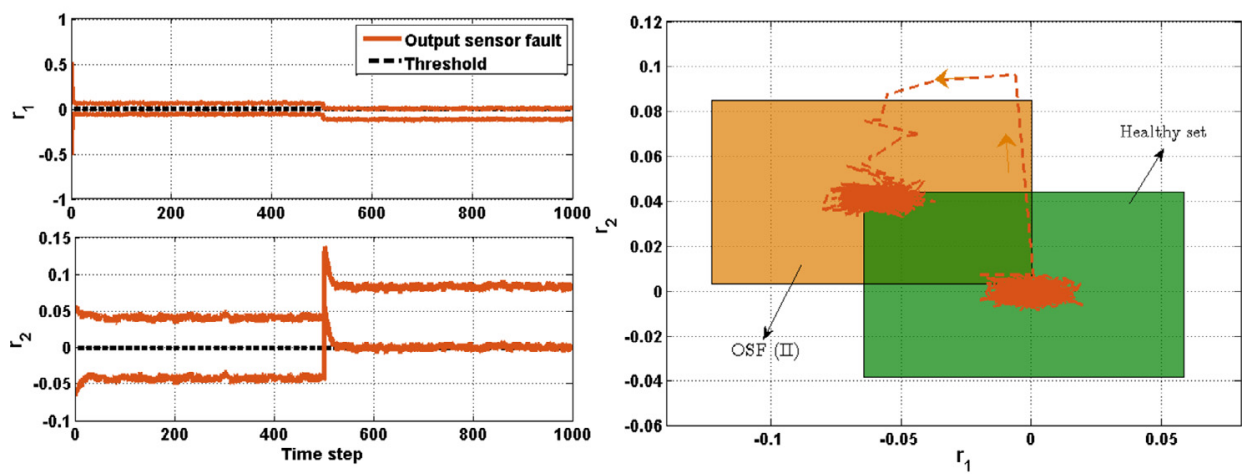

(b) $f_{y_{2}}=0.0080 \mathrm{~V}$ at $k=500$.

Fig. 8. FD results in the case of occurrence of the sensor fault $\left(f_{y_{2}}\right)$ during steady state, i.e., $k=500$.

that the fault with this magnitude is not detectable. Thus, Figs. 7 and 8 confirm through the simulation the obtained results previously presented in Eq. (69).

The last simulation that is considered for the case study is related to the case of input sensor fault. As mentioned before, the input sensor fault is a type of the fault that the input of the observer is influenced by the fault (see Eq. (24)). The simulation of the input sensor fault is carried out through the matrix $F_{u}$ and the vector $f_{u}$, i,e.,

$F_{u}=5, \quad f_{u}=f_{u}$,

where matrix $F_{u}$ is defined with the whole range of the input which is between $[0,5] \mathrm{V}$. Furthermore, $f_{u}$ denotes the effect of the fault influencing observer input. Then, the minimum magnitude of the input sensor fault is computed using Theorem 4.3 as

$f_{\min _{u}}^{\text {Det }}= \pm 0.6620 \mathrm{~V}$.

Similar to the actuator and output sensor faults, the MDF in the case of input sensor fault is obtained during steady-state operation of the system. Then, regarding the FD performance in this case, the fault is simulated at $k=500$ to illustrate the steady state. Furthermore, the following scenarios are considered for the implementation of the FD test:

- scenario (i): $f_{u}=0.6640 \mathrm{~V}$, slightly bigger than the magnitude $f_{\min _{u}}^{\text {Det }}= \pm 0.6620 \mathrm{~V}$, 

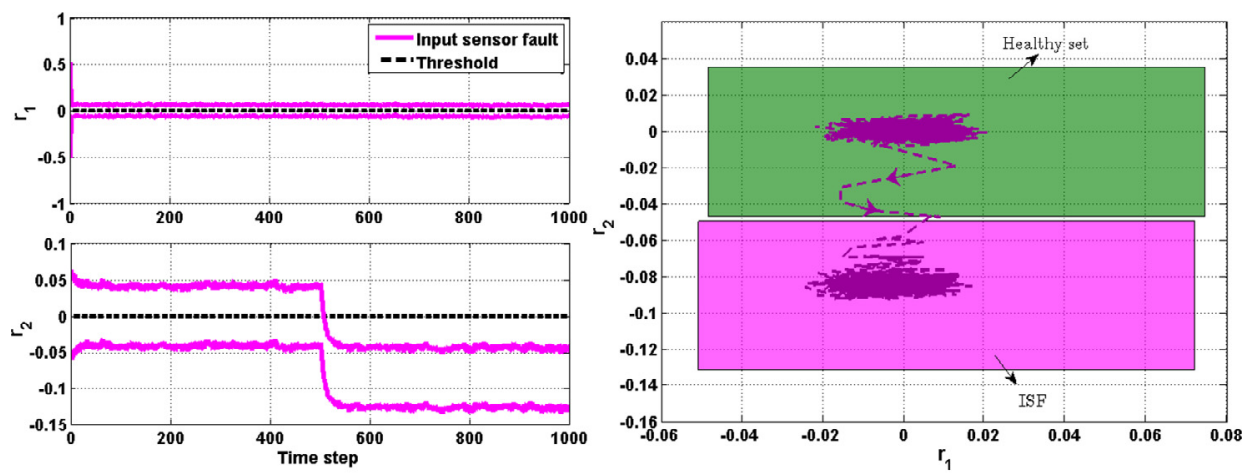

(a) $f_{u}=0.6640 \mathrm{~V}$ at $k=500$.
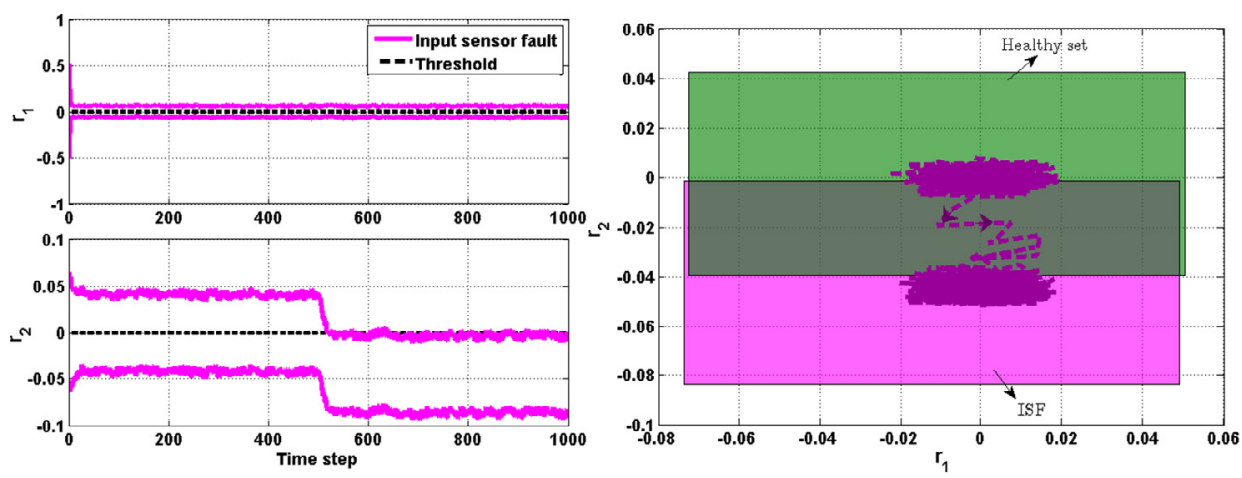

(b) $f_{u}=0.6600 \mathrm{~V}$ at $k=500$.

Fig. 9. FD results in the case of occurrence of the input sensor fault during steady state, i.e., $k=500$.

- scenario (ii): $f_{u}=0.6600 \mathrm{~V}$, slightly smaller than the magnitude $f_{\min _{u}}^{\text {Det }}= \pm 0.6620 \mathrm{~V}$.

Fig. 9 shows the FD test considering the mentioned scenarios during steady state for the case of input sensor fault. As can be seen in Fig. 9, considering the input sensor fault with bigger magnitude reported in (71), the separation of the healthy and faulty residual sets is obtained. Thus, the existence of the fault is proved. Also, the obtained overlap between the healthy and faulty residual sets when considering output sensor fault with smaller magnitude is presented in Fig. 9 corresponding to the magnitude that cannot be detected in steady state.

Further analysis is done by projecting the residual set into each residual space. It can be seen from the left side of the Fig. 9, when the first scenario is simulated, zero is out of the area between the upper and lower bounds of the residual set. Then, the fault will be detected. On the other hand, when the second scenario is simulated, zero is inside of the area between the upper and lower bounds of the residual set. Then, the fault cannot be detected.

Furthermore, Table 1 summarizes the obtained MDF in all the cases (actuator and sensor faults) using Theorem 4.3. Furthermore, those magnitudes of the fault that can be still detected at the end of the simulation, i.e., in steady state is obtained using the simulation and reported in Table 1.

From Table 1, the magnitude of the fault that can be detected considering the whole time range of the simulation is almost the same as the one obtained based on the theoretical 
Table 1

MDF during steady-state operation of the system.

\begin{tabular}{|c|c|c|c|c|}
\hline & \multirow{2}{*}{$\begin{array}{l}\text { Actuator fault } \\
f_{\min _{a}}^{\text {Det }} \\
\end{array}$} & \multicolumn{2}{|c|}{ Output sensor fault } & \multirow{2}{*}{$\frac{\text { Input sensor fault }}{f_{\min _{u}}^{\text {Det }}[\mathrm{V}]}$} \\
\hline & & $f_{\min _{y_{1}}}^{\text {Det }}[\mathrm{V}]$ & $f_{\min _{y_{2}}}^{\text {Det }}[\mathrm{V}]$ & \\
\hline Theoretical & \pm 0.3310 & \pm 0.2575 & \pm 0.0082 & \pm 0.6620 \\
\hline Simulation & \pm 0.3200 & \pm 0.2900 & \pm 0.0078 & \pm 0.6900 \\
\hline
\end{tabular}

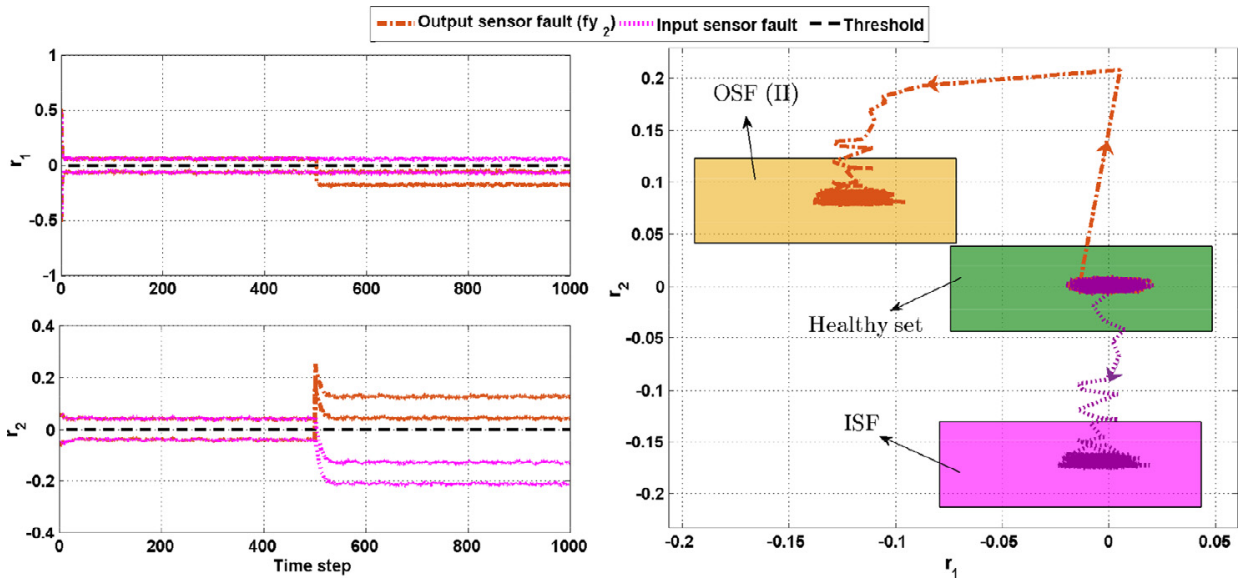

Fig. 10. Always separable (ISF and OSF (II) denote the residual set when simulation input sensor fault $f_{u}$ and output sensor fault $f_{y_{2}}$, respectively).

approach. However, in the case of sensor fault and due to the re-injection of the fault involved by the observer structure leading to some transient behavior (see the overshoot in Figs. 7 and 8), the magnitude of the detectable fault is improved on the whole time range of the simulation compared to steady state only.

As it is mentioned before, the fault with the magnitude obtained using Theorem 4.3 is only related to the detectability analysis and this magnitude of the fault is not valid in the case of isolability analysis. After detecting the fault by means of obtaining the separated sets in healthy and fault operation of the system, the isolation of the fault depends on satisfaction of the conditions in Theorem 4.2. In this regard, the magnitude of the different faults that are obtained using MIF analysis in Theorem 4.4 for the case study are reported on Table 2.

It can be seen from the results that are presented in Table 2, there are some cases that do not exist in the considered case study. These cases are denoted by - in Table 2. Furthermore, there are some other cases that the faults always can be isolable if they are larger than the MDF magnitude. These cases are shown by \# in Table 2. A possible explanation for having \# might be related to the direction of the fault in the case study that always there are some faults with the different directions, e.g., minimum isolable input sensor fault with respect to the output sensor fault. In these cases, after obtaining the separation between the healthy and faulty sets, the faulty sets are also separated. Fig. 10 illustrates one of the cases that always can be isolable with MDF magnitude since the direction of the faults are always different for the considered case study. 


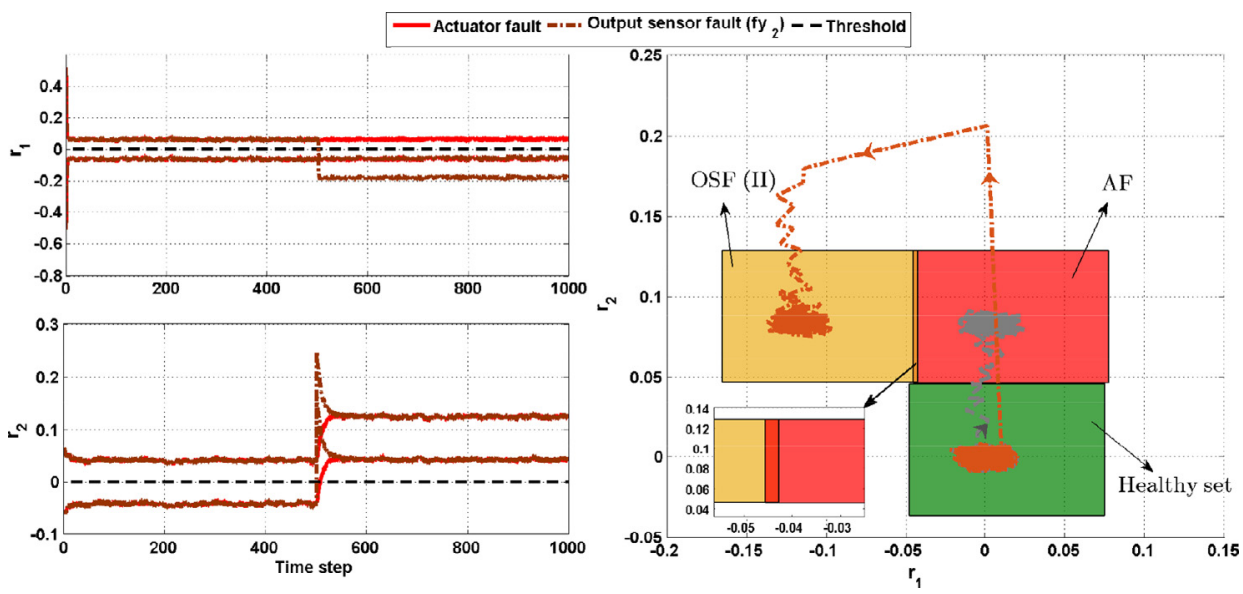

Fig. 11. FD results when the obtained magnitude using MDF is considered for the case of occurrence of $f_{y_{2}}$ and $f_{a}$ at the same time (AF denotes the residual set when simulation actuator fault $f_{a}$ ).

The implementation of the fault is done using the magnitudes that are obtained based on Theorem 4.3 and reported in Table 1 as $f_{u}=0.6640 \mathrm{~V}$ that is slightly bigger than the magnitude $f_{\min _{u}}^{\mathrm{Det}}= \pm 0.6620 \mathrm{~V}$ for the case of occurrence of $f_{u}$ and $f_{y_{2}}=0.0084 \mathrm{~V}$ that is slightly bigger than the magnitude $f_{\min _{y_{2}}}^{\text {Det }}= \pm 0.0082 \mathrm{~V}$ for the case of occurrence of $f_{y_{2}}$. As can be seen in Fig. 10, the occurrence of the input sensor fault $f_{u}$ and output sensor fault $f_{y_{2}}$ are always separable by the magnitude that is obtained based on Theorem 4.3.

However, the overlap can be obtained between the faulty residual sets in some cases implementing the fault with the obtained magnitude in Table 1. Fig. 11 illustrates one of the cases, e.g., the case of occurrence $f_{y_{2}}$ and $f_{a}$ at the same time.

In this case, the fault magnitudes are considered $f_{a}=0.3330$ that is slightly bigger than $f_{\min _{a}}^{\text {Det }}= \pm 0.3310$ and $f_{y_{2}}=0.0 .0084 \mathrm{~V}$ that is slightly bigger than $f_{\min _{y_{2}}}^{\text {Det }}= \pm 0.0082 \mathrm{~V}$ for the case of occurrence of $f_{a}$ and $f_{y_{2}}$, respectively. Fig. 11 shows that the separation between the faulty sets cannot be obtained when the occurrence of the faults are simulated with the magnitudes reported in Table 1.

Then, the magnitudes that are obtained in Table 2 are implemented in the simulation for the considered cases: $f_{y_{2}}=0.009 \mathrm{~V}$ and $f_{a}=0.35$. Therefore, as can be seen in Fig. 12 , the fault with the magnitude using Table 2 can be not only detectable, but also, can be isolable since the intersection between the faulty residual sets are obtained empty and the faulty sets are separated from each other and the healthy residual set.

Further analysis is done based on the simulation of the case of occurrence of all type of possible faults for the case study by considering slightly bigger magnitudes than the maximum MIF for the faults obtained in Table 2 as

$$
\begin{aligned}
f_{a} & =0.35, \\
f_{y_{1}} & =0.29 \mathrm{~V}, \\
f_{y_{2}} & =0.0095 \mathrm{~V}, \\
f_{u} & =0.73 \mathrm{~V} .
\end{aligned}
$$




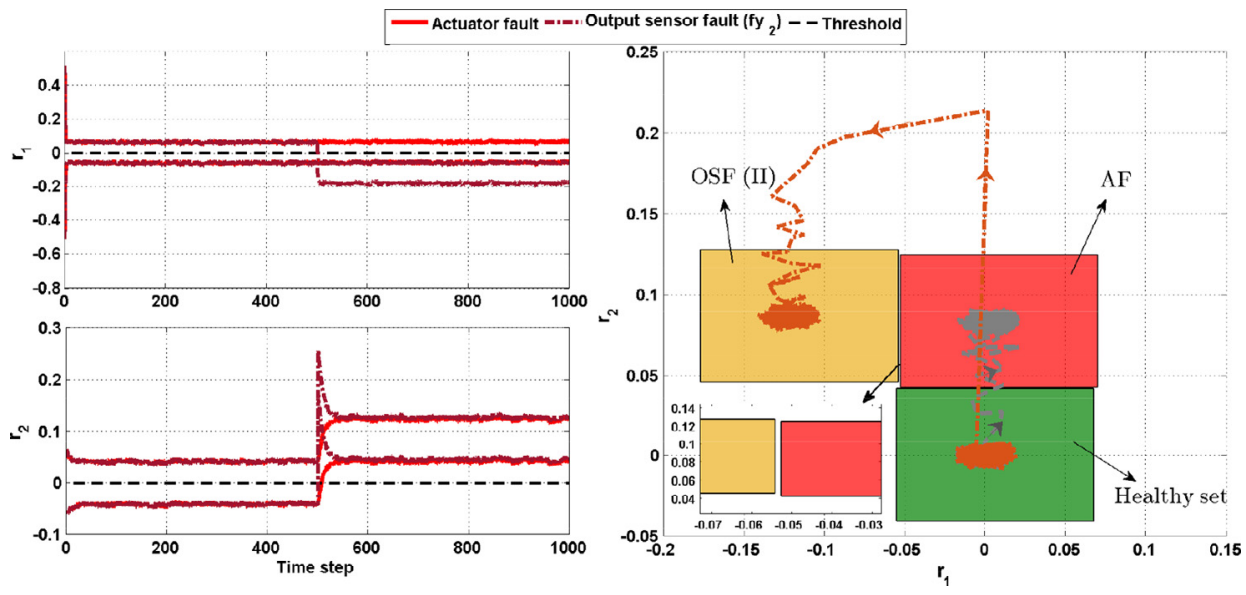

Fig. 12. FD results when the obtained magnitude using MIF is considered.

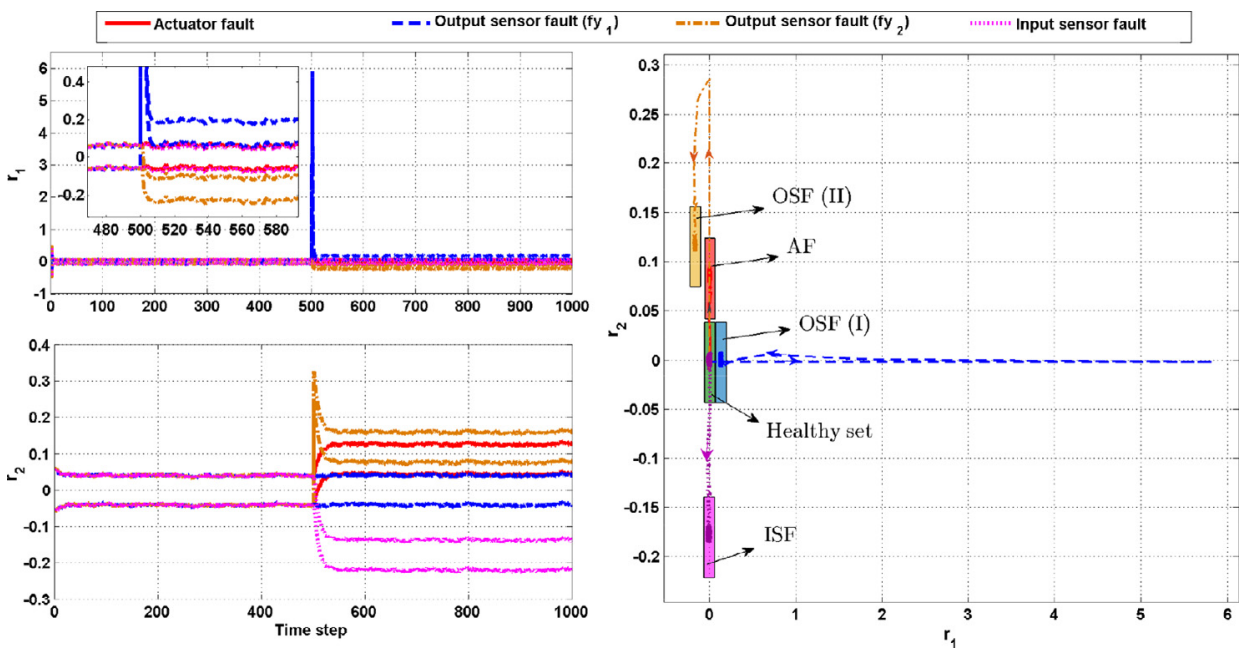

Fig. 13. FD results when the maximum magnitude that obtained using MIF analysis in Table 2 for each type of faults is simulated.

Table 2

MIF during steady-state operation of the system.

\begin{tabular}{lllll}
\hline & $f_{a}$ & $f_{y_{1}}[\mathrm{~V}]$ & $f_{y_{2}}[\mathrm{~V}]$ & $f_{u}[\mathrm{~V}]$ \\
\hline$f_{\min _{a}}^{\text {Iso }}$ & - & $\#$ & $\#$ & \pm 0.3460 \\
$f_{\min _{y_{1}}}^{\text {Iso }}$ & \pm 0.2808 & - & $\#$ & \pm 0.2691 \\
$f_{\min _{y_{2}}}^{\text {Iso }}$ & \pm 0.0089403 & \pm 0.008571 & - & \pm 0.0085701 \\
$f_{\min _{u}}^{\text {Iso }}$ & \pm 0.7219 & $\#$ & $\#$ & - \\
\hline
\end{tabular}

Figure 13 presents the case of occurrence of the fault with the maximum value obtained in Table 2, all type of faults are perfectly separated. This case correspond to a fault magnitudes that properly detectable and isolable. 


\section{Conclusions}

This paper has proposed a zonotopic interval observer-based Fault Detection and Isolation (FDI) algorithm integrated with the set-invariance approach. As a novelty, in the proposed FDI design, fault detectability and fault isolability can be guaranteed in both transient and steady states. The influences of all possible state disturbance and measurement noise are addressed using the zonotopic-set representation of a set. Furthermore, Minimum Detectable Fault (MDF) and Minimum Isolable Fault (MIF) have been characterized based on the sensitivity analysis integrated with set-invariance approach. Finally, a case study based on two-tank system is used to illustrate the obtained results. As a future research, the effect of the observer gain and the influence of the input over the state-bounding observer will be further analyzed in order to improve the algorithm for enhancing the sensitivity to the fault with respect to the influence of disturbance with the goal of improving the FDI performance. Moreover, the proposed FDI approach will be extended to deal with the case of multiple simultaneous faults.

\section{Acknowledgments}

This work has been partially funded by the Spanish State Research Agency (AEI) and the European Regional Development Fund (ERFD) through the projects DEOCS (ref. MINECO DPI2016-76493) and SCAV (ref. MINECO DPI2017-88403-R). This work has also been partially funded by AGAUR of Generalitat de Catalunya through the Advanced Control Systems (SAC) group grant (2017 SGR 482) and by Agència de Gestió d'Ajuts Universitaris i de Recerca.

\section{Appendix}

Definition 1 (Vector s-norm). The $s$-norm of a vector $\|x\|_{s}$ for $s=1,2, \ldots, n$ is defined as

$$
\|x\|_{s}=\left(\sum_{j=1}^{n}\left|x_{j}\right|^{s}\right)^{\frac{1}{s}} .
$$

Definition 2 (Zonotope). A zonotope $\left\langle c_{z}, R_{z}\right\rangle \subset \mathbb{R}^{n}$ with the center $c \in \mathbb{R}^{n}$ and the generator matrix $R \in \mathbb{R}^{n \times p}$ is a polytopic set defined as a linear image of the unit hypercube $[-1,1]^{n}$ :

$$
\left\langle c_{z}, R_{z}\right\rangle=\left\{c_{z}+R_{z} s,\|s\|_{\infty} \leq 1\right\} .
$$

Moreover, a centered zonotope is denoted by $\left\langle R_{z}\right\rangle=\left\langle 0, R_{z}\right\rangle$. Any permutation of the columns of $R$ leaves it invariant.

Definition 3 (Minkowski sum). Considering two sets $\mathcal{A}$ and $\mathcal{B}$, their Minkowski sum is a set defined as $\mathcal{A} \oplus \mathcal{B}=\{a+b \mid a \in \mathcal{A}, b \in \mathcal{B}\}$. Furthermore, the Minkowski sum of the zonotopes $\mathcal{Z}_{1}=\left\langle c_{z_{1}}, R_{z_{1}}\right\rangle$ and $\mathcal{Z}_{2}=\left\langle c_{z_{2}}, R_{z_{2}}\right\rangle$ is $\mathcal{Z}_{1} \oplus \mathcal{Z}_{2}=\left\langle c_{z_{1}}+c_{z_{2}},\left[R_{z_{1}}, \quad R_{z_{2}}\right]\right\rangle$.

Definition 4 (Interval hull). Interval hull of a given zonotope $\mathcal{Z}=\left\langle c_{z}, R_{z}\right\rangle$ is the smallest interval box that contains $\mathcal{Z}$ and it is denoted by $\mathcal{Z}$.

Definition 5 (Zonotope support strip). given zonotope $\mathcal{Z}=\left\langle c_{z}, R_{z}\right\rangle$ and a vector $C$, the zonotope support strip is introduced by $\mathscr{S}=z: s_{d} \leq C_{i} z \leq s_{u}$, where $i$ denotes the $i$-row of the 
vector $C$. Furthermore, $s_{d}$ and $s_{u}$ should satisfy $s_{d}=\min _{z \in \mathcal{Z}} C_{i} z$ and $s_{u}=\max _{z \in \mathcal{Z}} C_{i} z$ and they can be computed as

$$
\begin{aligned}
& s_{d}=C_{i} c_{z}+\left\|C_{i} R_{z}^{\top}\right\|_{1}, \\
& s_{u}=C_{i} c_{z}-\left\|C_{i} R_{z}^{\top}\right\|_{1} .
\end{aligned}
$$

Definition 6 (Invariant set). The invariant set $\Omega \subseteq \mathcal{Z}$ is the set which its existence allowed the evolution of a constrained system, where $z_{0} \in \Omega \subseteq \mathcal{Z}$ and then, $z_{K} \in \Omega \subseteq \mathcal{Z}$ for all time steps $k$.

Property 1 (Linear image). The linear image of a zonotope $\mathcal{Z}=\langle c, R\rangle$ by a compatible matrix $L$ is $L \odot\langle c, R\rangle=\langle L c, L R\rangle$.

Property 2 (Reduction operator). A reduction operator denoted $\downarrow_{q}$ permits to reduce the number of generators of a zonotope $\langle c, R\rangle$ to a fixed number $q$ while preserving the inclusion property $\langle c, R\rangle \subset\left\langle c, \downarrow_{q}\{R\}\right\rangle$. A simple yet efficient solution to compute $\downarrow_{q}\{R\}$ is given in [46]. It consists in sorting the columns of $R$ on decreasing Euclidean norm and enclosing the influence of the smaller columns only into an easily computable interval hull, so that the resulting matrix $\downarrow_{q}\{R\}$ has no more than $q$ columns.

Property 3 (Zonotope inclusion). Given a zonotope $\mathcal{Z}=\langle c, R\rangle \subset \mathbb{R}^{n}$, with a vector $c \in$ $\mathbb{R}^{n}$ denoting the center and an interval matrix $R \in \mathbb{R}^{n \times m}(n \leq m)$ denoting the shape of the zonotope, a zonotope inclusion indicated by $\diamond(\mathcal{Z})$ is defined as $\diamond(\mathcal{Z})=\langle c,[\operatorname{mid}(R), \quad S]\rangle$, where $S$ is a diagonal matrix that satisfies $S_{i i}=\sum_{j=1}^{m} \frac{\operatorname{diam}\left(R_{i j}\right)}{2}, i=1,2, \ldots, n$, with mid(.) and diam(.) are the center and diameter of interval matrix, respectively.

Property 4 (State zonotope inclusion). Given $\mathcal{X}_{k+1}=A \mathcal{X}_{k} \oplus B u_{k}$, where $A$ and $B$ are interval matrices and $u_{k}$ is the input at time instant $k$, considering $\mathcal{X}_{k}$ as a zonotope with the center $c_{x, k}$ and the shape matrix $R_{x, k}$ such $\mathcal{X}_{k}=\left\langle c_{x, k}, R_{x, k}\right\rangle$, the zonotopic state at the next time instant $k+1$ defined as $\mathcal{X}_{k+1}$ is bounded by a zonotope $\mathcal{X}_{k+1}^{e}=\left\langle c_{x, k+1}, R_{x, k+1}\right\rangle$, with

$c_{x, k+1}=\operatorname{mid}(A) c_{x, k}+\operatorname{mid}(B) u_{k}$,

$R_{x, k+1}=\left[\diamond\left(A R_{x, k}\right), \quad \frac{\operatorname{diam}(A)}{2} c_{x, k}, \quad \frac{\operatorname{diam}(B)}{2} u_{k}\right]$,

where $\diamond\left(A R_{x, k}\right)$ shows the shape matrix of the state bounding zonotope.

\section{References}

[1] F.K. Pour, V. Puig, C. Ocampo-Martinez, Multi-layer health-aware economic predictive control of a pasteurization pilot plant, Int. J. Appl. Math. Comput. Sci. 28 (1) (2018) 97-110.

[2] R. Isermann, P. Balle, Trends in the application of model-based fault detection and diagnosis of technical processes, Control Eng. Pract. 5 (5) (1997) 709-719.

[3] J. Gertler, Fault Detection and Diagnosis in Engineering Systems, CRC Press, USA, 1998.

[4] J. Cai, H. Ferdowsi, J. Sarangapani, Model-based fault detection, estimation, and prediction for a class of linear distributed parameter systems, Automatica 66 (2016) 122-131.

[5] V. Puig, Fault diagnosis and fault tolerant control using set-membership approaches: Application to real case studies, Int. J. Appl. Math. Comput. Sci. 20 (4) (2010) 619-635.

[6] J. Chen, R.J. Patton, Robust Model-Based Fault Diagnosis for Dynamic Systems, Kluwer Academic Press, USA, 1999. 
[7] M. Blanke, M. Kinnaert, J. Lunze, M. Staroswiecki, J. Schröder, Diagnosis and Fault-Tolerant Control, 2, Springer, USA, 2006.

[8] V. Puig, S. Montes de Oca, J. Blesa, Adaptive threshold generation in robust fault detection using interval models: time-domain and frequency-domain approaches, Int. J. Adapt. Control Signal Process. 27 (10) (2013) 873-901.

[9] T. Alamo, J.M. Bravo, E.F. Camacho, Guaranteed state estimation by zonotopes, Automatica 41 (6) (2005) 1035-1043.

[10] T. Raïssi, D. Efimov, A. Zolghadri, Interval state estimation for a class of nonlinear systems, IEEE Trans. Autom. Control 57 (1) (2012) 260-265.

[11] R.E. Kalman, et al., A new approach to linear filtering and prediction problems, J. Basic Eng. 82 (1) (1960) 35-45.

[12] P.S. Maybeck, Stochastic Models, Estimation, and Control, 3, Academic Press, USA, 1982.

[13] F. Schweppe, Recursive state estimation: Unknown but bounded errors and system inputs, IEEE Trans. Autom. Control 13 (1) (1968) 22-28.

[14] L. Uusitalo, A. Lehikoinen, I. Helle, K. Myrberg, An overview of methods to evaluate uncertainty of deterministic models in decision support, Environ. Model. Softw. 63 (2015) 24-31.

[15] G.M. Ziegler, Lectures on Polytopes (Graduate Texts in Mathematics), Springer, USA, 1994.

[16] C. Combastel, Zonotopes and kalman observers: gain optimality under distinct uncertainty paradigms and robust convergence, Automatica 55 (2015) 265-273.

[17] M. Pourasghar, V. Puig, C. Ocampo-Martinez, Comparison of set-membership and interval observer approaches for state estimation of uncertain systems, in: Proceedings of the European Control Conference (ECC), Denmark, 2016, pp. 1111-1116.

[18] M. Pourasghar, V. Puig, C. Ocampo-Martinez, Interval observer versus set-membership approaches for fault detection in uncertain systems using zonotopes, Int. J. Robust Nonlinear Control 29 (10) (2019) $2819-2843$.

[19] W. Kühn, Rigorously computed orbits of dynamical systems without the wrapping effect, Computing 61 (1) (1998) 47-67.

[20] M. Pourasghar, C. Combastel, V. Puig, C. Ocampo-Martinez, FD-ZKF: A zonotopic Kalman filter optimizing fault detection rather than state estimation, J. Process Control 73 (2019) 89-102.

[21] J. Meseguer, V. Puig, T. Escobet, Robust fault detection linear interval observers avoiding the wrapping effect, IFAC Proc. Vol. 41 (2) (2008) 11570-11575.

[22] V. Puig, J. Quevedo, T. Escobet, A. Stancu, Robust fault detection using linear interval observers, IFAC Proc. Vol. 36 (5) (2003) 579-584.

[23] F. Stoican, M. Hovd, S. Olaru, Explicit invariant approximation of the mRPI set for LTI dynamics with zonotopic disturbances, in: Proceedings of the 52nd Annual Conference on Decision and Control (CDC), Italy, 2013, pp. 3237-3242.

[24] J.C.L. Chan, C.P. Tan, H. Trinh, Robust fault reconstruction for a class of infinitely unobservable descriptor systems, Int. J. Syst. Sci. 48 (8) (2017) 1646-1655.

[25] G. Rinaldi, P.P. Menon, C. Edwards, A. Ferrara, Sliding mode based dynamic state estimation for synchronous generators in power systems, IEEE Control Syst. Lett. 2 (4) (2018) 785-790.

[26] W. Reinelt, A. Garulli, L. Ljung, Comparing different approaches to model error modeling in robust identification, Automatica 38 (5) (2002) 787-803.

[27] M.A. Medina, D. Theilliol, D. Sauter, Simultaneous fault diagnosis and robust activation function estimation of linear multiple-models considering the bounded modelling uncertainty, in: Proceedings of the International Symposium on Mediterranean Conference on Control and Automation, IEEE, 2005, pp. 405-410.

[28] F. Blanchini, S. Miani, Set-Theoretic Methods in Control, Springer, USA, 2008.

[29] F. Stoican, S. Olaru, J.A. De Doná, M.M. Seron, Zonotopic ultimate bounds for linear systems with bounded disturbances, IFAC Proc. Vol. 44 (1) (2011) 9224-9229.

[30] E. Kofman, Non-conservative ultimate bound estimation in LTI perturbed systems, Automatica 41 (10) (2005) 1835-1838.

[31] E. Kofman, H. Haimovich, M.M. Seron, A systematic method to obtain ultimate bounds for perturbed systems, Int. J. Control 80 (2) (2007) 167-178.

[32] M.M. Seron, X.W. Zhuo, J.A. De Doná, J.J. Martinez, Multisensor switching control strategy with fault tolerance guarantees, Automatica 44 (1) (2008) 88-97.

[33] C. Ocampo-Martinez, J.A. De Doná, M. Seron, Actuator fault-tolerant control based on set separation, Int. J. Adapt. Control. Signal Process. 24 (12) (2010) 1070-1090. 
[34] M.M. Seron, J.A. De Doná, Actuator fault tolerant multi-controller scheme using set separation based diagnosis, Int. J. Control 83 (11) (2010) 2328-2339.

[35] F. Xu, F. Stoican, V. Puig, C. Ocampo-Martinez, S. Olaru, On the relationship between interval observers and invariant sets in fault detection, in: Proceedings of the Conference on Control and Fault-Tolerant Systems (SysTol), France, 2013, pp. 49-54.

[36] S.V. Rakovic, E.C. Kerrigan, K.I. Kouramas, D.Q. Mayne, Invariant approximations of the minimal robust positively invariant set, IEEE Trans. Autom. Control 50 (3) (2005) 406-410.

[37] Z. Artstein, S.V. Raković, Feedback and invariance under uncertainty via set-iterates, Automatica 44 (2) (2008) $520-525$.

[38] M. Pourasghar, V. Puig, C. Ocampo-Martinez, Interval observer-based fault detectability analysis using mixed set-invariance theory and sensitivity analysis approach, Int. J. Syst. Sci. 50 (3) (2019) 495-516.

[39] M. Pourasghar, V. Puig, C. Ocampo-Martinez, Characterization of the minimum detectable fault of interval observers by using set-invariance theory, in: Proceedings of the 3rd Conference on Control and Fault-Tolerant Systems (SysTol), Spain, 2016, pp. 79-86.

[40] A.R. Kodakkadan, M. Pourasghar, V. Puig, S. Olaru, C. Ocampo-Martinez, V. Reppa, Observer-based sensor fault detectability: about robust positive invariance approach and residual sensitivity, in: Proceedings of the 20th World Congress of the International Federation of Automatic Control (IFAC), France, 2017.

[41] T. Dang, Approximate reachability computation for polynomial systems, in: Proceedings of the Hybrid Systems: Computation and Control (HSCC), 6, Springer, USA, 2006, pp. 138-152.

[42] F. Blanchini, Set invariance in control, Automatica 35 (11) (1999) 1747-1767.

[43] J.C.L. Chan, C.P. Tan, H. Trinh, M.A.S. Kamal, State and fault estimation for a class of non-infinitely observable descriptor systems using two sliding mode observers in cascade, J. Frankl. Inst. 356 (5) (2019a) 3010-3029.

[44] J.C.L. Chan, C.P. Tan, H. Trinh, M.A.S. Kamal, Y.S. Chiew, Robust fault reconstruction for a class of non-infinitely observable descriptor systems using two sliding mode observers in cascade, Appl. Math. Comput. 350 (2019b) 78-92.

[45] K.H. Johansson, The quadruple-tank process: A multivariable laboratory process with an adjustable zero, IEEE Trans. Control Syst. Technol. 8 (3) (2000) 456-465.

[46] C. Combastel, A state bounding observer based on zonotopes, in: Proceedings of the European Control Conference (ECC), UK, 2003, pp. 2589-2594. 